\title{
On Analytical Solution of Time-Fractional Biological Population Model by means of Generalized Integral Transform with Their Uniqueness and Convergence Analysis
}

\author{
Saima Rashid $\mathbb{D}^{\mathbb{D}},{ }^{1}$ Rehana Ashraf, ${ }^{2}$ and Ebenezer Bonyah $\mathbb{i}^{3,4}$ \\ ${ }^{1}$ Department of Mathematics, Government College University, Faisalabad 38000, Pakistan \\ ${ }^{2}$ Department of Mathematics, Lahore College for Women University, 54000 Lahore, Pakistan \\ ${ }^{3}$ Department of Mathematics Education, Akenten Appiah-Menka University of Skills Training and Entrepreneurial Development, \\ Kumasi, Ghana \\ ${ }^{4}$ Department of Mathematics, Faculty of Science and Technology, Universitas Airlangga, Surabaya 60115, Indonesia
}

Correspondence should be addressed to Ebenezer Bonyah; ebbonya@gmail.com

Received 8 September 2021; Accepted 3 December 2021; Published 27 February 2022

Academic Editor: Azhar Hussain

Copyright (C) 2022 Saima Rashid et al. This is an open access article distributed under the Creative Commons Attribution License, which permits unrestricted use, distribution, and reproduction in any medium, provided the original work is properly cited.

\begin{abstract}
This research utilizes the generalized integral transform and the Adomian decomposition method to derive a fascinating explicit pattern for outcomes of the biological population model (BPM). It assists us in comprehending the dynamical technique of demographic variations in BPMs and generates significant projections. Besides that, generalized integral transforms are the unification of other existing transforms. To investigate the closed form solutions, we employed a fractional complex transform to deal with a partial differential equation of fractional order and a generalized decomposition method was applied to analyze the nonlinear equation. Several aspects of the Caputo and Atangana-Baleanu fractional derivative operators are discussed with the aid of a generalized integral transform. In mathematical terms, the variety of equations and their solutions have been discovered and identified with various novel features of the projected model. To provide additional context for these ideas, numerous sorts of illustrations and tabulations are presented. The precision and efficacy of the proposed technique suggest that it can be used for a variety of nonlinear evolutionary problems.
\end{abstract}

\section{Introduction}

Historically, a framework of nonlinear developmental equations was designed to model the proportion of a demographic in particular domains $[1,2]$. Several scholars have examined analytical, semiautomatic, and numerical solutions to fractional systems in a variety of fields, including virology, chaos, bifurcation, thermodynamics, neural networks, random walks, image processing, aquifer and anomalous spreading, and so on [3-5]. These behaviors have been described by expounding fractional numerical simulations compared with experimental findings in order to assert their nonlocal features, when this sort of characteristic [6-10] cannot be articulated using nonlinear PDEs of integer order.
Numerous efficient and comprehensive approaches, such as the tan-cot function method [11], the Adomian decomposition method [12], the homotopy perturbation method [13], the homotopy analysis method [14], the wavelet method [15], and the Lie symmetry analysis [16], have been constructed determined by the flexibility to form complex nonlinear phenomena in diversified disciplines such as diseases, optical fibers, fluid flow, thermodynamics, electrostatics, reaction-diffusion, and plasma physics.

Population dynamics simulations are being used to comprehend, interpret, and forecast the movements and permanence of biodiversity. Such frameworks are used to evaluate a population's welfare, evaluate the reasons for population decreases or rapid expansion, recommend corporate agendas, and assess the forecasting of a population's 
anticipated reactions to diverse organizational processes from a managerial perspective.

Leading up to delving into the step-by-step methodologies for formulating and interpreting continuously BPMs, we will take a look at population genetic sculpting in the past, granting us an overview of the key figures in the field of ecology and evolution as well as the techniques they formed to comprehend population systems from a physics perspective.

In this study, we investigate a fundamental model in biology. The degenerate parabolic equation appears in the spatial diffusion of biological populations $[17,18]$ :

$$
Q_{\varsigma}=Q_{\mathbf{w}_{1} \mathbf{w}_{1}}^{2}+Q_{\mathbf{w}_{2} \mathbf{w}_{2}}^{2}+\sigma(Q), \quad \varsigma \geq 0, \mathbf{w}_{1}, \mathbf{w}_{2} \in \mathbb{R},
$$

subject to initial condition (IC) $Q\left(\mathbf{w}_{1}, \mathbf{w}_{2}, 0\right)$, where $Q$ signifies the population density and $\sigma$ denotes the population supply due to births and deaths.

Certain specific features of (1), for instance, Hölder estimates of its solutions, are investigated in [19]. Furthermore, two basic examples of constitutive formulations for $\sigma$ are the Malthusian law [17],

$$
\sigma=\nu \mathbb{Q}, \quad(\nu=\text { constant })
$$

and the Verhulst law [19],

$$
\sigma=\nu Q-\gamma Q^{2}, \quad(\nu, \gamma=\text { constant })
$$

Thus, we assume the more generic version of $\sigma$ as $\sigma(\mathbb{Q})=$ $\hbar Q^{\alpha}\left(1-r_{1} Q^{\beta}\right)$ which is held for porous media $[20,21]$ as follows:

$Q_{\zeta}=Q_{\mathbf{w}_{1} \mathbf{w}_{1}}^{2}+Q_{\mathbf{w}_{2} \mathbf{w}_{2}}^{2}+\hbar Q^{\alpha}\left(1-r_{1} Q^{\beta}\right), \quad \varsigma \geq 0, \mathbf{w}_{1}, \mathbf{w}_{2} \in \mathbb{R}$,

where $\alpha, \beta, \hbar$, and $r_{1}$ are real constants.

It is noted that Malthusian and Verhulst laws are the particular cases that can be attained by inserting $\hbar=\nu, \alpha=$ 1 , and $r_{1}=0 \quad$ and $\quad \hbar=\nu, \alpha=\beta=1$, and $r_{1}=\gamma / \nu$, respectively.

FDEs are remarkably appropriate for simulating biochemical mechanisms because they are specifically apprehensive about biological analytical models' memory, which appears to be a substantial advancement over traditional integer-order mathematical methodologies, and it is associated with chaotic systems, which are prevalent in BPMs. Rashid et al. [22] recently considered the fractional spatial diffusion of a BPM via a new integral transform in the singular and nonsingular kernel settings. Zellal and Belghaba [23] reported the variational iteration method to find an accurate algorithm for solving BPM. Singh [24] expounded the analysis of the fractional blood alcohol model with a composite fractional derivative. An epidemic model $S E I_{1} I_{2} R$ for the transmission of $\mathrm{HIV}$ epidemics by the mean value theorem was investigated by Naik et al. [25]. A homotopy decomposition method was employed by investigating the HIV infection of $\mathrm{C} 4^{+}$by Atangana and Alabaraoye [26]. For more details on BPM, see $[27,28]$ and the citations therein.

Amidst George Adomian's massive boost in 1980, the Adomian decomposition method introduced a well-noted terminology. It has been intensively implemented for a diverse set of nonlinear PDEs, for instance, the Korteweg-De Vries model [29], Fisher's model [30], Zakharov-Kuznetsov equation [31], and so on. The ADM was determined to be significantly related to a variety of integral transforms, including Laplace, Swai, Mohand, Aboodh, Elzaki, and others. Very recently, Jafari [32] propounded a well-known integral transform which is known to generalized integral transform. The dominant feature of this transformation is that it has the ability to recapture several existing transformations (see Remark 1).

Motivated by the above propensity, we aim to establish a semianalytical approach by mingling the generalized integral transform with the Adomian decomposition method. With the assistance of fractional derivative operators, we constructed the approximate analytical solution to BPM. Furthermore, the convergence and uniqueness analysis is carried out in the Caputo fractional derivative framework. The proposed findings are in close harmony with the exact solutions. Sketching and comparison analysis solutions are drafted with a powerful and pragmatic approach. Both operators consistently behave according to the projected method.

\section{Preliminaries}

In this section, we evoke some essential concepts, notions, and definitions concerning fractional derivative operators depending on power and Mittag-Leffler as a kernel, along with the detailed consequences of the generalized integral transform.

Definition 1 (see [6]). The Caputo fractional derivative (CFD) is described as follows:

${ }_{0}^{c} \mathbf{D}_{\varsigma}^{\chi}= \begin{cases}\frac{1}{\Gamma(r-\chi)} \int_{0}^{\varsigma} \frac{\widehat{Q}^{(r)}\left(w_{1}\right)}{\left(\varsigma-\mathbf{w}_{1}\right)^{\chi+1-r}} \mathrm{~d} \mathbf{w}_{1}, & r-1<\chi<r, \\ \frac{\mathrm{d}^{r}}{\mathrm{~d} \varsigma^{r}} Q(\varsigma), & \chi=r .\end{cases}$

Definition 2 (see [7]). The Atangana-Baleanu fractional derivative operator in the Caputo form $(\mathrm{ABC})$ is stated as follows:

$$
{ }_{\eta_{1}}^{\mathrm{ABC}} \mathbf{D}_{\zeta}^{\chi}(\mathscr{Q}(\varsigma))=\frac{\mathbb{A}(\chi)}{1-\chi} \int_{\eta_{1}}^{\varsigma} \mathscr{Q}^{\prime}(\varsigma) E_{\chi}\left[-\frac{\chi\left(\varsigma-w_{1}\right)^{\chi}}{1-\chi}\right] \mathrm{d} \mathbf{w}_{1},
$$

where $Q \mathcal{Q} \in \mathscr{H}^{1}\left(a_{1}, a_{2}\right)$ (Sobolev space), $a_{1}<a_{2}, \chi \in[0,1]$, and $\mathbb{A}(\chi)$ signifies a normalization function as $\mathbb{A}(\chi)=$ $\mathbb{A}(0)=\mathbb{A}(1)=1$.

Definition 3 (see [7]). The fractional integral of the ABC operator is described as follows:

$$
\underset{\eta_{1}}{\mathrm{ABC}} \mathscr{J}_{\varsigma}^{\chi}(\mathscr{Q}(\varsigma))=\frac{1-\chi}{\mathbb{A}(\chi)} \mathscr{Q}(\varsigma)+\frac{\chi}{\Gamma(\chi) \mathbb{A}(\chi)} \int_{\eta_{1}}^{\varsigma} \mathscr{Q}\left(w_{1}\right)\left(\varsigma-w_{1}\right)^{\chi-1} \mathrm{~d} \mathbf{w}_{1} .
$$


Definition 4 (see [32]). Consider an integrable mapping Q (५) defined on a set $\mathscr{P}$; then,

$\mathscr{P}=\{\mathscr{Q}(\varsigma): \exists M>0, \kappa>0,|\mathbb{Q}(\varsigma)|<M \exp (\kappa \varsigma), \quad$ if $\varsigma \geq 0\}$.

Definition 5 (see [32]). Suppose the mappings $\phi(\mathfrak{g}), \psi(\mathfrak{g}): \mathbb{R}^{+} \mapsto \mathbb{R}^{+}$such that $\varphi(\mathfrak{g}) \neq 0 \forall \mathfrak{g} \in \mathbb{R}^{+}$. The generalized integral transform of the mapping $Q(\varsigma)$ presented by $\mathbf{Q}(\mathfrak{g})$ is described as

$$
\mathbb{J}\{\mathfrak{Q}(\varsigma), \mathfrak{g}\}=\mathbf{Q}(\mathfrak{I})=\phi\left(s_{1}\right) \int_{0}^{\infty} \mathscr{Q}(\varsigma) \exp (-\psi(\mathfrak{g}) \varsigma) \mathrm{d} \varsigma
$$

Theorem 1 (see [32]) (convolution property). For generalized integral transform, the subsequent holds true:

$$
\mathbb{J}\left\{\mathcal{Q}_{1} * \mathbb{Q}_{2}\right\}=\frac{1}{\phi(\mathfrak{g})} \mathbf{Q}_{1}(\mathfrak{g}) * \mathbf{Q}_{2}(\mathfrak{g})
$$

Definition 6. The generalized integral transform of the CFD operator is stated as follows:

$$
\begin{aligned}
\mathbb{J}\left\{{ }_{0}^{c} \mathbf{D}_{\zeta}^{\chi}(\mathcal{Q}(\varsigma)), \mathfrak{g}\right\}= & \psi^{\chi}(\mathfrak{g}) \mathbf{Q}\left(s_{1}\right)-\phi(\mathfrak{g}) \sum_{\kappa=0}^{\chi-1} \psi^{\chi-\kappa-1}\left(s_{1}\right) \mathbb{Q}^{(\kappa)}(0), \\
& r-1<\chi<r, \phi, \psi>0 .
\end{aligned}
$$

Remark 1. Definition 6 leads to the following conclusions:

(1) Taking $\phi(\mathfrak{g})=1$ and $\psi(\mathfrak{g})=\mathfrak{g}$, then we acquire the Laplace transform [33]

(2) Taking $\phi(\mathfrak{g})(1 / \mathfrak{g})$ and $\psi(\mathfrak{g})=(1 / \mathfrak{g})$, then we acquire the $\alpha$-Laplace transform [34]

(3) Taking $\phi(\mathfrak{g})=(1 / \mathfrak{g})$ and $\psi(\mathfrak{g})=(1 / \mathfrak{s})$, then we acquire the Sumudu transform [35]

(4) Taking $\phi(\mathfrak{g})=(1 / \mathfrak{g})$ and $\psi(\mathfrak{g})=1$, then we acquire the Aboodh transform [36]

(5) Taking $\phi(\mathfrak{g})=\mathfrak{g}$ and $\psi(\mathfrak{g})=\mathfrak{g}^{2}$, then we acquire the Pourreza transform [37, 38]

(6) Taking $\phi(\mathfrak{g})=\mathfrak{g}$ and $\psi(\mathfrak{g})=(1 / \mathfrak{g})$, then we acquire the Elzaki transform [39]

(7) Taking $\phi(\mathfrak{g})=\mathbf{w}_{2}$ and $\psi(\mathfrak{g})=\left(\mathfrak{g} / \mathbf{w}_{2}\right)$, then we acquire the natural transform [40]

(8) Taking $\phi(\mathfrak{s})=\mathfrak{g}^{2}$ and $\psi(\mathfrak{g})=\mathfrak{g}$, then we acquire the Mohand transform [41]

(9) Taking $\phi(\mathfrak{g})=\left(1 / \mathfrak{g}^{2}\right)$ and $\psi(\mathfrak{g})=(1 / \mathfrak{g})$, then we acquire the Swai transform [42]

(10) Taking $\phi(\mathfrak{g})=1$ and $\psi(\mathfrak{g})=(1 / \mathfrak{g})$, then we get the Kamal transform [43]

(11) Taking $\phi(\mathfrak{g})=\mathfrak{g}^{\alpha}$ and $\psi(\mathfrak{g})=(1 / \mathfrak{g})$, then we acquire the $G_{-}$transform $[44,45]$
Definition 7 (see [46]). The generalized integral transform of the ABC fractional derivative operator is described as

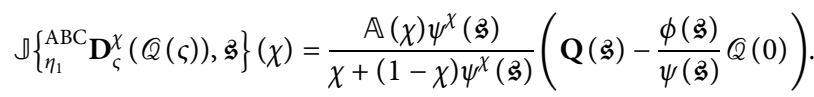

Remark 2. Definition 7 leads to the following conclusions:

(1) Taking $\phi(\mathfrak{g})=1$ and $\psi(\mathfrak{g})=\mathfrak{g}$, then we acquire the Laplace transform of $\mathrm{ABC}$ fractional derivative operator $[7,47]$

(2) Taking $\phi(\mathfrak{s})=\mathfrak{g}$ and $\psi(\mathfrak{s})=(1 / \mathfrak{g})$, then we acquire the Elzaki transform of ABC fractional derivative operator [48]

(3) Taking $\phi(\mathfrak{g})=\psi(\mathfrak{g})=(1 / \mathfrak{s})$, then we get the Sumudu transform of ABC fractional derivative operator [49]

(4) Taking $\phi(\mathfrak{g})=1$ and $\psi(\mathfrak{g})=\mathfrak{g} / \mathbf{w}_{2}$, then we get the Shehu transform of ABC fractional derivative operator [49]

Definition 8 (see [50]). The Mittag-Leffler function for single parameter is described as

$$
E_{\chi}(z)=\sum_{\kappa=0}^{\infty} \frac{z_{1}^{\kappa}}{\Gamma(\kappa \chi+1)}, \quad \chi, z_{1} \in \mathbb{C}, \mathfrak{R}(\chi) \geq 0 .
$$

\section{Description of the Generalized Decomposition Method}

Consider the generic fractional form of PDE:

$$
\begin{aligned}
& \mathbf{D}_{\varsigma}^{\chi} Q\left(w_{1}, \varsigma\right)+\mathscr{L} Q\left(\mathbf{w}_{1}, \varsigma\right)+\tilde{N} Q\left(\mathbf{w}_{1}, \varsigma\right) \\
& =\mathscr{F}\left(\mathbf{w}_{1}, \varsigma\right), \quad \varsigma>0,0<\chi \leq 1,
\end{aligned}
$$

with ICs

$$
\mathcal{Q}\left(\mathbf{w}_{1}, 0\right)=\mathscr{G}\left(\mathbf{w}_{1}\right)
$$

where $\mathbf{D}_{\varsigma}^{\chi}=\left(\partial^{\chi} Q\left(\mathbf{w}_{1}, \varsigma\right) / \partial \varsigma^{\chi}\right)$ symbolizes the Caputo and ABC fractional derivative of order $\chi \in(0,1]$, while $\mathfrak{Q}$ and $\tilde{N}$ denote the linear and nonlinear factors, respectively. Also, $\mathscr{F}\left(\mathbf{w}_{1}, \varsigma\right)$ represents the source term.

Taking into account the generalized integral transform to (14), we acquire

$\mathbb{J}\left[\mathbf{D}_{\varsigma}^{\chi} \mathscr{Q}\left(\mathbf{w}_{1}, \varsigma\right)+\mathscr{L} \mathbb{Q}\left(\mathbf{w}_{1}, \varsigma\right)+\tilde{N} \mathscr{Q}\left(\mathbf{w}_{1}, \varsigma\right)\right]=\mathbb{J}\left[\mathscr{F}\left(\mathbf{w}_{1}, \varsigma\right)\right]$.

First, by applying the differentiation rule of generalized integral transform with respect to $\mathrm{CFD}$, we apply the $\mathrm{ABC}$ fractional derivative operator as follows: 


$$
\begin{aligned}
& \psi^{\chi}(\mathfrak{g}) \mathscr{U}\left(\mathbf{w}_{1}, \mathfrak{s}\right)=\phi(\mathfrak{g}) \sum_{\kappa=0}^{\ell-1} \psi^{\chi-1-\kappa}(\mathfrak{g}) \mathscr{Q}^{(\kappa)}(0)+\mathbb{J}\left[\mathscr{L} \mathscr{Q}\left(\mathbf{w}_{1}, \varsigma\right)+\widetilde{N} \mathscr{Q}\left(\mathbf{w}_{1}, \varsigma\right)\right]+\mathbb{J}\left[\mathscr{F}\left(\mathbf{w}_{1}, \varsigma\right)\right], \\
& \frac{\psi^{\chi}(\mathfrak{g}) \mathbb{A}(\chi)}{\chi+(1-\chi) \psi^{\chi}(\mathfrak{I})} \mathscr{U}\left(\mathbf{w}_{1}, \mathfrak{g}\right)=\frac{\phi(\mathfrak{g})}{\psi(\mathfrak{I})} \frac{\psi^{\chi}(\mathfrak{g}) \mathbb{A}(\chi)}{\chi+(1-\chi) \psi^{\chi}(\mathfrak{I})} \mathscr{Q}(0)+\mathbb{J}\left[\mathscr{L} \mathscr{Q}\left(\mathbf{w}_{1}, \varsigma\right)+\tilde{N} \mathscr{Q}\left(\mathbf{w}_{1}, \varsigma\right)\right]+\mathbb{J}\left[\mathscr{F}\left(\mathbf{w}_{1}, \varsigma\right)\right]
\end{aligned}
$$

The inverse generalized integral transform of (17) and (18) yields

$$
\begin{aligned}
& \mathscr{Q}\left(\mathbf{w}_{1}, \varsigma\right)=\mathbb{J}^{-1}\left[\phi(\mathfrak{g}) \sum_{\kappa=0}^{\ell-1} \psi(\mathfrak{g})^{\chi-\kappa-1} \mathscr{Q}^{(\kappa)}(0)+\frac{1}{\psi^{\chi}(\mathfrak{g})} \mathbb{J}\left[\mathscr{F}\left(\mathbf{w}_{1}, \varsigma\right)\right]\right]-\mathbb{J}^{-1}\left[\frac{1}{\psi^{\chi}(\mathfrak{I})} \mathbb{J}\left[\mathscr{L} \mathscr{Q}\left(\mathbf{w}_{1}, \varsigma\right)+\tilde{N} \mathscr{Q}\left(\mathbf{w}_{1}, \varsigma\right)\right]\right] \\
& \mathscr{Q}\left(\mathbf{w}_{1}, \varsigma\right)=\mathbb{J}^{-1}\left[\frac{\phi(\mathfrak{I})}{\psi(\mathfrak{I})} \mathfrak{Q}(0)+\frac{\chi+(1-\chi) \psi^{\chi}(\mathfrak{g})}{\psi^{\chi}(\mathfrak{I}) \mathbb{A}(\chi)} \mathbb{J}\left[\mathscr{F}\left(\mathbf{w}_{1}, \varsigma\right)\right]\right]-\mathbb{J}^{-1}\left[\frac{\chi+(1-\chi) \psi^{\chi}(\mathfrak{g})}{\psi^{\chi}(\mathfrak{I}) \mathbb{A}(\chi)} \mathbb{I}\left[\mathscr{L} \mathscr{Q}\left(\mathbf{w}_{1}, \varsigma\right)+\widetilde{N} \mathscr{Q}\left(\mathbf{w}_{1}, \varsigma\right)\right]\right] .
\end{aligned}
$$

The generalized decomposition method solution where $\mathbb{Q}\left(\mathbf{w}_{1}, \varsigma\right)$ is represented by the following infinite series:

$$
Q\left(\mathbf{w}_{1}, \varsigma\right)=\sum_{\ell=0}^{\infty} Q_{\ell}\left(\mathbf{w}_{1}, \varsigma\right)
$$

Thus, the nonlinear term $\widetilde{N}\left(\mathbf{w}_{1}, \varsigma\right)$ can be evaluated by the Adomian decomposition method prescribed as

$$
\widetilde{N} Q\left(\mathbf{w}_{1}, \varsigma\right)=\sum_{\ell=0}^{\infty} \widetilde{A}_{\ell}\left(\mathscr{Q}_{0}, Q_{1}, \ldots\right), \quad \ell=0,1, \ldots,
$$

$$
\widetilde{A}_{\ell}\left(\mathscr{Q}_{0}, \mathscr{Q}_{1}, \ldots\right)=\frac{1}{\ell !}\left[\frac{\mathrm{d}^{\ell}}{\mathrm{d} \chi^{\ell}} \widetilde{N}\left(\sum_{j=0}^{\infty} \chi^{j} \mathscr{Q}_{j}\right)\right]_{\chi=0}, \quad \ell>0 .
$$

Inserting (21) and (22) into (19) and (20), respectively, we have

$$
\begin{aligned}
& \sum_{\ell=0}^{\infty} Q_{\ell}\left(\mathbf{w}_{1}, \varsigma\right)=\mathscr{G}\left(\mathbf{w}_{1}\right)+\widetilde{\mathscr{G}}\left(\mathbf{w}_{1}\right)-\mathbb{J}^{-1}\left[\frac{1}{\psi^{\chi}(\mathfrak{I})} \sqrt{ }\left[\mathscr{L} \mathscr{Q}\left(\mathbf{w}_{1}, \varsigma\right)+\sum_{\ell=0}^{\infty} \widetilde{A}_{\ell}\right]\right] \\
& \sum_{\ell=0}^{\infty} \mathbb{Q}_{\ell}\left(\mathbf{w}_{1}, \varsigma\right)=\mathscr{G}\left(\mathbf{w}_{1}\right)+\tilde{\mathscr{G}}\left(\mathbf{w}_{1}\right)-\mathbb{J}^{-1}\left[\frac{\chi+(1-\chi) \psi^{\chi}(\mathfrak{S})}{\mathbb{A}(\chi) \psi^{\chi}(\mathfrak{S})} \rrbracket\left[\mathscr{L} \mathscr{Q}\left(\mathbf{w}_{1}, \varsigma\right)+\sum_{\ell=0}^{\infty} \widetilde{A}_{\ell}\right]\right] .
\end{aligned}
$$

Consequently, the recursive technique for (24) and (25) is established as follows:

$$
\begin{aligned}
Q_{0}\left(\mathbf{w}_{1}, \varsigma\right) & =\mathscr{G}\left(\mathbf{w}_{1}\right)+\tilde{G}\left(\mathbf{w}_{1}\right), \quad \ell=0 \\
\mathbb{Q}_{\ell+1}\left(\mathbf{w}_{1}, \varsigma\right) & =-\coprod^{-1}\left[\frac{1}{\psi^{\chi}(\mathfrak{g})} \rrbracket\left[\mathfrak{L}\left(\mathscr{Q}_{\ell}\left(\mathbf{w}_{1}, \varsigma\right)\right)+\sum_{\ell=0}^{\infty} \widetilde{A}_{\ell}\right]\right], \quad \ell \geq 1, \\
\mathbb{Q}_{\ell+1}\left(\mathbf{w}_{1}, \varsigma\right) & =-\coprod^{-1}\left[\frac{\chi+(1-\chi) \psi^{\chi}(\mathfrak{g})}{\mathbb{A}(\chi) \psi^{\chi}(\mathfrak{g})} \rrbracket\left[\mathfrak{L}\left(\mathbb{Q}_{\ell}\left(\mathbf{w}_{1}, \varsigma\right)\right)+\sum_{\ell=0}^{\infty} \widetilde{A}_{\ell}\right]\right], \quad \ell \geq 1 .
\end{aligned}
$$




\section{Some New Mathematical Aspects of Generalized Decomposition Method}

The subsequent subsections will highlight how the sufficient requirements guarantee the emergence of a unique solution. Our anticipated existence of solutions in the case of GDM is followed by [51].
Theorem 2 (uniqueness theorem). Equation (26) has a unique solution whenever $0<\epsilon<1$, where $\epsilon=\left(\left(\mathscr{K}_{1}+\mathscr{K}_{2}+\mathscr{K}_{3}\right)\right) \varsigma^{(\chi)} /(\Gamma(\chi+1))$.

Proof. Assume all continuous functions on the Banach space are denoted by $\Omega=(\mathbb{C}[\mathscr{I}],\|\cdot\|)$. Also, suppose that $\mathscr{I}=$ $[0, \mathscr{T}]$ has the norm $\|\cdot\|$. Now, we define a function $\mathcal{U}: \Omega \mapsto \Omega$ such that

$$
\mathcal{Q}_{\ell+1}\left(\mathbf{w}_{1}, \varsigma\right)=\mathscr{Q}\left(\mathbf{w}_{1}, \varsigma\right)+\mathbb{J}^{-1}\left[\frac{1}{\psi^{\chi}(\mathfrak{G})} \mathbb{D}\left[\mathfrak{Q}\left[\mathscr{Q}_{\ell}\left(\mathbf{w}_{1}, \varsigma\right)\right]+\bar{P}\left[\mathscr{Q}_{\ell}\left(\mathbf{w}_{1}, \varsigma\right)\right]+\tilde{N}\left[\mathscr{Q}_{\ell}\left(\mathbf{w}_{1}, \varsigma\right)\right]\right]\right], \quad \ell \geq 0
$$

where $\mathbb{Q}\left[Q\left(\mathbf{w}_{1}, \varsigma\right)\right] \equiv \partial^{3} \mathscr{Q}\left(\mathbf{w}_{1}, \varsigma\right) / \partial \mathbf{w}_{1}^{2}$ and $\bar{P}\left[Q\left(\mathbf{w}_{1}, \varsigma\right)\right] \equiv$ $\partial Q\left(\mathbf{w}_{1}, \varsigma\right) / \partial \mathbf{w}_{1}$. Here, suppose that $\mathbb{Q}\left[\mathbb{Q}\left(\mathbf{w}_{1}, \varsigma\right)\right]$ and $\mathfrak{M}\left[Q\left(\mathbf{w}_{1}, \varsigma\right)\right]$ are also Lipschitzian with
$|\bar{P} Q-\bar{P} \widehat{Q}|<\mathscr{K}_{1}|\mathscr{Q}-\widehat{Q}| \quad$ and $\quad|\mathscr{L} Q-\mathfrak{Q} \widehat{Q}|<\mathscr{K}_{2}|\mathscr{Q}-\widehat{Q}|$, where $\mathscr{K}_{1}$ and $\mathscr{K}_{2}$ are Lipschitz constant, respectively, and $\widehat{Q}$ and $\widehat{Q}$ are distinct functional values.

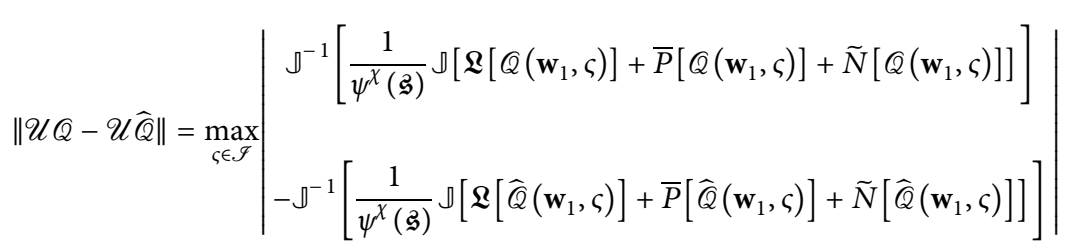

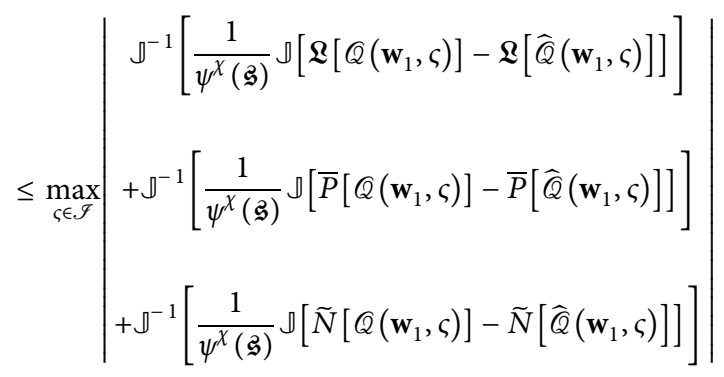

$$
\begin{aligned}
& \leq \max _{\varsigma \in \mathscr{I}}\left[\begin{array}{c}
\mathscr{K}_{1} \mathbb{J}^{-1}\left[\frac{1}{\psi^{\chi}(\mathfrak{I})} \mathbb{J}\left|\mathscr{Q}\left(\mathbf{w}_{1}, \varsigma\right)-\widehat{Q}\left(\mathbf{w}_{1}, \varsigma\right)\right|\right] \\
+\mathscr{K}_{2} \mathbb{J}^{-1}\left[\frac{1}{\psi^{\chi}(\mathfrak{G})} \mathbb{J}\left|\mathscr{Q}\left(\mathbf{w}_{1}, \varsigma\right)-\widehat{\mathbb{Q}}\left(\mathbf{w}_{1}, \varsigma\right)\right|\right] \\
+\mathscr{K}_{3} \mathbb{J}^{-1}\left[\frac{1}{\psi^{\chi}(\mathfrak{I})} \mathbb{J}\left|\mathbb{Q}\left(\mathbf{w}_{1}, \varsigma\right)-\widehat{\mathbb{Q}}\left(\mathbf{w}_{1}, \varsigma\right)\right|\right]
\end{array}\right] \\
& \leq \max _{\varsigma \in \mathscr{I}}\left(\mathscr{K}_{1}+\mathscr{K}_{2}+\mathscr{K}_{3}\right) \mathbb{J}^{-1}\left[\frac{1}{\psi^{\chi}(\mathfrak{g})} \mathbb{J}\left|\mathscr{Q}\left(\mathbf{w}_{1}, \varsigma\right)-\widehat{\mathscr{Q}}\left(\mathbf{w}_{1}, \varsigma\right)\right|\right] \\
& \leq\left(\mathscr{K}_{1}+\mathscr{K}_{2}+\mathscr{K}_{3}\right) \mathbb{J}^{-1}\left[\frac{1}{\psi^{\chi+1}(\mathfrak{g})} \rrbracket\left\|\mathscr{Q}\left(\mathbf{w}_{1}, \varsigma\right)-\widehat{Q}\left(\mathbf{w}_{1}, \varsigma\right)\right\|\right] \\
& =\left(\mathscr{K}_{1}+\mathscr{K}_{2}+\mathscr{K}_{3}\right) \mathbb{J}^{-1}\left[\frac{\phi(\mathfrak{g})}{\psi^{X}(\mathfrak{g})}\left\|\mathscr{Q}\left(\mathbf{w}_{1}, \varsigma\right)-\widehat{Q}\left(\mathbf{w}_{1}, \varsigma\right)\right\|\right] \\
& =\frac{\left(\left(\mathscr{K}_{1}+\mathscr{K}_{2}+\mathscr{K}_{3}\right)\right) \varsigma^{(\chi)}}{\Gamma(\chi+1)}\left\|\mathscr{Q}\left(\mathbf{w}_{1}, \varsigma\right)-\widehat{Q}\left(\mathbf{w}_{1}, \varsigma\right)\right\| .
\end{aligned}
$$


Since $0<\epsilon<1$, the mapping is contraction. Consequently, by Banach contraction fixed point theorem, (14) has a unique solution. This gives the desired result.

Theorem 3 (convergence analysis). The general form solution of (14) will be convergent.

Proof. Assume that $\widehat{W}_{\ell}$ be the $n$th partial sum, i.e., $\widehat{W}_{\ell}=\sum_{m=0}^{\ell} Q_{\ell}\left(\mathbf{w}_{1}, \varsigma\right)$. Here, we prove a Cauchy sequence $\left\{\widehat{W}_{\ell}\right\}$ in Banach space $U$.
We acquire by considering a new form of Adomian polynomials:

$$
\begin{aligned}
& \bar{R}\left(\widehat{W}_{\ell}\right)=\tilde{\mathscr{H}}_{\ell}+\sum_{p=0}^{\ell-1} \widetilde{\mathscr{H}}_{p}, \\
& \tilde{N}\left(\widehat{W}_{\ell}\right)=\tilde{\mathscr{H}}_{\ell}+\sum_{c=0}^{\ell-1} \tilde{\mathscr{H}}_{c} .
\end{aligned}
$$

Now,

$$
\begin{aligned}
& \left\|\widehat{W}_{\ell}-\widehat{W}_{q}\right\|=\max _{\zeta \in \mathscr{I}}\left|\widehat{W}_{\ell}-\widehat{W}_{q}\right| \\
& =\max _{\varsigma \in \mathcal{F}}\left|\sum_{m=q+1}^{\ell} \widehat{\mathbb{Q}}\left(\mathbf{w}_{1}, \varsigma\right)\right|, \quad(m=1,2,3, \ldots) \\
& \leq \max _{\varsigma \in \mathcal{J}}\left|\begin{array}{c}
\mathbb{J}^{-1}\left[\frac{1}{\psi^{X}(\mathfrak{I})} \mathbb{J}\left[\sum_{m=q+1}^{\ell} \mathfrak{Q}\left[\mathbb{Q}_{\ell-1}\left(\mathbf{w}_{1}, \varsigma\right)\right]\right]\right] \\
+\mathbb{J}^{-1}\left[\frac{1}{\psi^{X}(\mathfrak{I})} \sqrt{\frac{1}{\psi^{X}(\mathfrak{g})}}\left[\sum_{m=q+1}^{\ell} \bar{P}\left[\sum_{m=q+1}^{\ell} \tilde{\mathscr{Q}}_{\ell-1}\left(\mathbf{w}_{1}, \varsigma\right)\right]\right]\right. \\
\left.\left.\left.\ell \mathbf{w}_{1}, \varsigma\right)\right]\right]
\end{array}\right| \\
& =\max _{\varsigma \in \mathcal{I}}\left|\begin{array}{c}
\mathbb{J}^{-1}\left[\frac{1}{\psi^{X}(\mathfrak{I})} \rrbracket\left[\sum_{m=q}^{\ell-1} \mathfrak{Q}\left[\mathbb{Q}_{\ell}\left(\mathbf{w}_{1}, \varsigma\right)\right]\right]\right. \\
+\mathbb{J}^{-1}\left[\frac{1}{\psi^{X}(\mathfrak{I})} \rrbracket\left[\sum_{m=q}^{\ell-1} \bar{P}\left[\mathscr{Q}_{\ell}\left(\mathbf{w}_{1}, \varsigma\right)\right]\right]\right] \\
+\mathbb{J}^{-1}\left[\frac{1}{\psi^{X}(\mathfrak{g})} \rrbracket\left[\sum_{m=q}^{\ell-1} \tilde{\mathscr{H}}_{\ell}\left(\mathbf{w}_{1}, \varsigma\right)\right]\right]
\end{array}\right|
\end{aligned}
$$

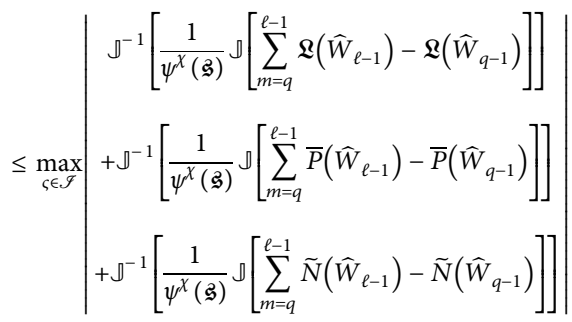

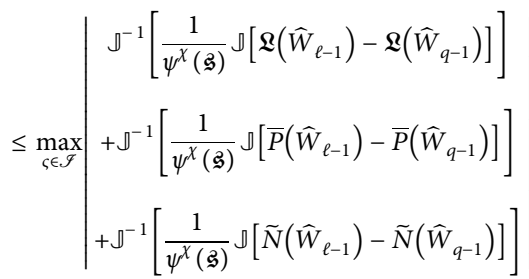

$$
\begin{aligned}
& \leq \mathscr{K}_{1} \max _{\zeta \in \mathscr{I}} \rrbracket^{-1}\left|\left[\frac{1}{\psi^{\chi}(\mathfrak{g})} \rrbracket\left[\left(\widehat{W}_{\ell-1}\right)-\left(\widehat{W}_{q-1}\right)\right]\right]\right| \\
& +\mathscr{K}_{2} \max _{\varsigma \in \mathscr{I}}\left|\mathbb{J}^{-1}\left[\frac{1}{\psi^{\chi}(\mathfrak{G})} \mathbb{J}\left[\left(\widehat{W}_{\ell-1}\right)-\left(\widehat{W}_{q-1}\right)\right]\right]\right| \\
& \left.+\mathscr{K}_{3} \max _{\varsigma \in \mathscr{I}} \mid \mathbb{J}^{-1}\left[\frac{1}{\psi^{\chi}(\mathfrak{G})} \sqrt{ }\right]\left[\left(\widehat{W}_{\ell-1}\right)-\left(\widehat{W}_{q-1}\right)\right]\right] \mid \\
& =\left(\mathscr{K}_{1}+\mathscr{K}_{2}+\mathscr{K}_{3}\right) \rrbracket^{-1}\left[\frac{\phi(\mathfrak{g})}{\psi^{X+1}(\mathfrak{g})}\left\|\widehat{W}_{\ell-1}-\widehat{W}_{q-1}\right\|\right. \\
& =\frac{\left(\mathscr{K}_{1}+\mathscr{K}_{2}+\mathscr{K}_{3}\right) \varsigma^{(\chi)}}{\Gamma(\chi+1)}\left\|\widehat{W}_{\ell-1}-\widehat{W}_{q-1}\right\| .
\end{aligned}
$$


Consider $n=q+1$; then,

$$
\left\|\widehat{W}_{q+1}-\widehat{W}_{q}\right\| \leq \epsilon\left\|\widehat{W}_{q}-\widehat{W}_{q-1}\right\| \leq \widehat{W}^{2}\left\|\widehat{W}_{q-1}-\widehat{W}_{q-2}\right\| \leq \cdots \leq \epsilon^{q}\left\|\widehat{W}_{1}-\widehat{W}_{0}\right\|,
$$

where $\left(\mathscr{K}_{1}+\mathscr{K}_{2}+\mathscr{K}_{3}\right) \varsigma^{(\chi)} /(\Gamma(\chi+1))$. Now, from triangular inequality, we have

$$
\begin{aligned}
\left\|\widehat{W}_{\ell}-\widehat{W}_{q}\right\| & \leq\left\|\widehat{W}_{q+1}-\widehat{W}_{q}\right\|+\left\|\widehat{W}_{q+2}-\widehat{W}_{q+1}\right\|+\cdots+\left\|\widehat{W}_{\ell}-\widehat{W}_{\ell-1}\right\| \\
& \leq\left[\epsilon^{q}+\epsilon^{q+1}+\cdots+\epsilon^{\ell-1}\right]\left\|\widehat{W}_{1}-\widehat{W}_{0}\right\| \\
& \leq \epsilon^{q}\left(\frac{1-\epsilon^{\ell-q}}{\epsilon}\right)\left\|Q_{1}\right\| .
\end{aligned}
$$

Since $0<\epsilon<1$, we have $\left(1-\epsilon^{\ell-q}\right)<1$; then,

$$
\left\|\widehat{W}_{\ell}-\widehat{W}_{q}\right\| \leq \frac{\epsilon^{q}}{1-\epsilon} \max _{\varsigma \in \mathscr{I}}\left\|\widehat{Q}_{1}\right\| .
$$

Therefore, $\left|Q_{1}\right|<\infty$ (since $Q\left(\mathbf{w}_{1}, \varsigma\right)$ is bounded). Furthermore, as $q \mapsto \infty$, then $\left\|\widehat{W}_{\ell}-\widehat{W}_{q}\right\| \mapsto 0$. Thus, $\left\{\widehat{W}_{1}\right\}$ is a Cauchy sequence in $K$. Consequently, the series $\sum_{n=0}^{\infty} \widehat{Q}_{\ell}$ is convergent and this yields the immediate consequence.

Theorem 4 (see [51]) (error estimate). The absolute error of the series solution (14)-(26) is calculated as

$$
\max _{\varsigma \in \mathscr{I}}\left|\mathcal{Q}\left(\mathbf{w}_{1}, \varsigma\right)-\sum_{\ell=1}^{q} \mathcal{Q}_{\ell}\left(\mathbf{w}_{1}, \varsigma\right)\right| \leq \frac{\epsilon^{q}}{1-\epsilon} \max _{\varsigma \in \mathscr{I}}\left\|Q_{1}\right\| .
$$

\section{Application of Generalized Integral Transform for Biological Population Model}

In what follows, we illustrate the technique described in Section 3 by considering three numerical tests to validate the supremacy and efficacy of the generalized decomposition method.

Example 1 (Malthusian law [17]). Assume the time-fractional BPM (4) having $\alpha=1$ and $r_{1}=0$; then,

$$
\mathbf{D}_{\varsigma}^{\alpha} Q\left(\mathbf{w}_{1}, \mathbf{w}_{2}, \varsigma\right)=Q_{\mathbf{w}_{1} \mathbf{w}_{1}}^{2}\left(\mathbf{w}_{1}, \mathbf{w}_{2}, \varsigma\right)+Q_{\mathbf{w}_{2} \mathbf{w}_{2}}^{2}\left(\mathbf{w}_{1}, \mathbf{w}_{2}, \varsigma\right)+\hbar Q\left(\mathbf{w}_{1}, \mathbf{w}_{2}, \varsigma\right)
$$

with IC

$$
Q_{0}\left(\mathbf{w}_{1}, \mathbf{w}_{2}, 0\right)=\sqrt{\mathbf{w}_{1}+\mathbf{w}_{2}+\mathbf{w}_{1} \mathbf{w}_{2}}
$$

Proof. Foremost, we provide the solution of (35) in two general cases.
Case 1First, we apply the Caputo fractional derivative operator coupled with the generalized integral transform and Adomian decomposition method. Applying the generalized integral transform for Example 1,

$$
\psi^{\chi}(\mathfrak{g}) \mathcal{U}\left(\mathbf{w}_{1}, \mathfrak{g}\right)-\phi(\mathfrak{g}) \sum_{\kappa=0}^{m-1} \psi^{\chi-\kappa-1}(\mathfrak{g}) Q^{(\kappa)}(0)=\mathbb{J}\left[\mathscr{Q}_{\mathbf{w}_{1} \mathbf{w}_{1}}^{2}\left(\mathbf{w}_{1}, \mathbf{w}_{2}, \varsigma\right)+\widehat{Q}_{\mathbf{w}_{2} \mathbf{w}_{2}}^{2}\left(\mathbf{w}_{1}, \mathbf{w}_{2}, \varsigma\right)+\hbar Q\left(\mathbf{w}_{1}, \mathbf{w}_{2}, \varsigma\right)\right]
$$

Taking into consideration the IC given in (36), we have 


$$
\mathcal{U}\left(\mathbf{w}_{1}, \mathfrak{g}\right)=\frac{\psi(\mathfrak{g})}{\phi(\mathfrak{g})} \mathscr{Q}\left(\mathbf{w}_{1}, \mathbf{w}_{2}, 0\right)+\frac{1}{\psi^{\chi}(\mathfrak{g})} \mathbb{J}\left[\mathcal{Q}_{\mathbf{w}_{1} \mathbf{w}_{1}}^{2}\left(\mathbf{w}_{1}, \mathbf{w}_{2}, \varsigma\right)+\mathbb{Q}_{\mathbf{w}_{2} \mathbf{w}_{2}}^{2}\left(\mathbf{w}_{1}, \mathbf{w}_{2}, \varsigma\right)+\hbar \mathbb{Q}\left(\mathbf{w}_{1}, \mathbf{w}_{2}, \varsigma\right)\right]
$$

Employing the inverse generalized integral transform, we obtain

$$
\mathcal{Q}\left(\mathbf{w}_{1}, \mathbf{w}_{2}, \varsigma\right)=\mathbb{J}^{-1}\left[\frac{\psi(\mathfrak{g})}{\phi(\mathfrak{g})} \mathcal{Q}\left(\mathbf{w}_{1}, \mathbf{w}_{2}, 0\right)+\frac{1}{\psi^{\chi}(\mathfrak{g})} \mathbb{J}\left[\mathcal{Q}_{\mathbf{w}_{1} \mathbf{w}_{1}}^{2}\left(\mathbf{w}_{1}, \mathbf{w}_{2}, \varsigma\right)+Q_{\mathbf{w}_{2} \mathbf{w}_{2}}^{2}\left(\mathbf{w}_{1}, \mathbf{w}_{2}, \varsigma\right)+\hbar \mathbb{Q}\left(\mathbf{w}_{1}, \mathbf{w}_{2}, \varsigma\right)\right]\right]
$$
find

Thanks to the generalized decomposition method, we

$$
\begin{aligned}
\mathcal{Q}_{0}\left(\mathbf{w}_{1}, \mathbf{w}_{2}, \varsigma\right) & =\mathbb{J}^{-1}\left[\frac{\psi(\mathfrak{I})}{\phi(\mathfrak{g})} \mathscr{Q}\left(\mathbf{w}_{1}, \mathbf{w}_{2}, 0\right)\right]=\mathbb{J}^{-1}\left[\frac{\psi(\mathfrak{g})}{\phi(\mathfrak{g})} \sqrt{\mathbf{w}_{1}+\mathbf{w}_{2}+\mathbf{w}_{1} \mathbf{w}_{2}}\right] \\
& =\sqrt{\mathbf{w}_{1}+\mathbf{w}_{2}+\mathbf{w}_{1} \mathbf{w}_{2}} .
\end{aligned}
$$

Here, we surmise that the unknown function $Q\left(\mathbf{w}_{1}, \mathbf{w}_{2}, \varsigma\right)$ can be written by an infinite series of the form

$$
\mathcal{Q}\left(\mathbf{w}_{1}, \mathbf{w}_{2}, \varsigma\right)=\sum_{\ell=0}^{\infty} Q_{\ell}\left(\mathbf{w}_{1}, \mathbf{w}_{2}, \varsigma\right)
$$

Also, the nonlinearity $\mathscr{F}(\mathbb{Q})$ can be decomposed by an infinite series of polynomials represented by

$$
\begin{aligned}
& \mathscr{F}(Q)=Q_{\mathbf{w}_{1} \mathbf{w}_{1}}^{2}=\sum_{\ell=0}^{\infty} \mathscr{A}_{\ell}, \\
& \mathscr{F}(Q)=Q_{\mathbf{w}_{2} \mathbf{w}_{2}}^{2}=\sum_{\ell=0}^{\infty} \mathscr{B}_{\ell},
\end{aligned}
$$

where $Q_{\ell}\left(\mathbf{w}_{1}, \mathbf{w}_{2}, \varsigma\right)$ will be evaluated recurrently and $\mathscr{A}_{\ell}$ and $\mathscr{B}_{\ell}$ are the so-called polynomials of $\mathbb{Q}_{0}, Q_{1}, \ldots, Q_{\ell}$ established by [52].

$$
\sum_{\ell=0}^{\infty} Q_{\ell+1}\left(\mathbf{w}_{1}, \mathbf{w}_{2}, \varsigma\right)=\mathbb{J}^{-1}\left[\frac{1}{\psi^{\chi}(\mathfrak{S})} \mathbb{J}\left[\sum_{\ell=0}^{\infty}(\mathscr{A})_{\ell}+\sum_{\ell=0}^{\infty}(\mathscr{B})_{\ell}+\hbar \sum_{\ell=0}^{\infty}(\mathscr{Q})_{\ell}\right]\right], \quad \ell=0,1,2, \ldots
$$
follows:

The first few Adomian polynomials are presented as

$$
\begin{aligned}
& \mathscr{A}_{\ell}\left(Q^{2}\right)_{\mathbf{w}_{1} \mathbf{w}_{1}}= \begin{cases}\left(Q_{0}^{2}\right)_{\mathbf{w}_{1} \mathbf{w}_{1}}, & \ell=0, \\
\left(2 Q_{0} Q_{1}\right)_{\mathbf{w}_{1} \mathbf{w}_{1}}, & \ell=1, \\
\left(Q_{1}^{2}+2 Q_{0} Q_{1}\right)_{\mathbf{w}_{1} \mathbf{w}_{1}}, & \ell=2,\end{cases} \\
& \mathscr{B}_{\ell}\left(Q^{2}\right)_{\mathbf{w}_{2} \mathbf{w}_{2}}= \begin{cases}\left(Q_{0}^{2}\right)_{\mathbf{w}_{2} \mathbf{w}_{2}}, & \ell=0, \\
\left(2 Q_{0} Q_{1}\right)_{\mathbf{w}_{2} \mathbf{w}_{2}}, & \ell=1, \\
\left(Q_{1}^{2}+2 Q_{0} Q_{1}\right)_{\mathbf{w}_{2} \mathbf{w}_{2}}, & \ell=2 .\end{cases}
\end{aligned}
$$

$$
\begin{aligned}
\mathbb{Q}_{1}\left(\mathbf{w}_{1}, \mathbf{w}_{2}, \varsigma\right) & =\mathbb{J}^{-1}\left[\frac{1}{\psi^{\chi}(\mathfrak{g})} \mathbb{J}\left[\mathscr{A}_{0}+\mathscr{B}_{0}+\hbar \mathscr{Q}_{0}\right]\right] \\
& =\hbar \sqrt{\mathbf{w}_{1}+\mathbf{w}_{2}+\mathbf{w}_{1} \mathbf{w}_{2}} \frac{\varsigma^{\chi}}{\Gamma(\chi+1)}, \\
\mathbb{Q}_{2}\left(\mathbf{w}_{1}, \mathbf{w}_{2}, \varsigma\right) & =\mathbb{J}^{-1}\left[\frac{1}{\psi^{\chi}(\mathfrak{G})} \mathbb{J}\left[\mathscr{A}_{1}+\mathscr{B}_{1}+\hbar \mathscr{Q}_{1}\right]\right] \\
& =\hbar^{2} \sqrt{\mathbf{w}_{1}+\mathbf{w}_{2}+\mathbf{w}_{1} \mathbf{w}_{2}} \frac{\varsigma^{2 \chi}}{\Gamma(2 \chi+1)}, \\
\mathbb{Q}_{3}\left(\mathbf{w}_{1}, \mathbf{w}_{2}, \varsigma\right) & =\mathbb{J}^{-1}\left[\frac{1}{\psi^{\chi}(\mathfrak{S})} \mathbb{J}\left[\mathscr{A}_{2}+\mathscr{B}_{2}+\hbar \mathscr{Q}_{2}\right]\right. \\
& =\hbar^{3} \sqrt{\mathbf{w}_{1}+\mathbf{w}_{2}+\mathbf{w}_{1} \mathbf{w}_{2}} \frac{\varsigma^{3 \chi}}{\Gamma(3 \chi+1)},
\end{aligned}
$$

For $\ell=0,1,2,3, \ldots$, 
The approximate solution for Example 1 is expressed as follows:

$$
\begin{aligned}
Q\left(\mathbf{w}_{1}, \mathbf{w}_{2}, \varsigma\right) & =\Phi_{0}\left(\mathbf{w}_{1}, \mathbf{w}_{2}, \varsigma\right)+\Phi_{1}\left(\mathbf{w}_{1}, \mathbf{w}_{2}, \varsigma\right)+\Phi_{2}\left(\mathbf{w}_{1}, \mathbf{w}_{2}, \varsigma\right)+\Phi_{3}\left(\mathbf{w}_{1}, \mathbf{w}_{2}, \varsigma\right)+\cdots \\
& =\sqrt{\mathbf{w}_{1}+\mathbf{w}_{2}+\mathbf{w}_{1} \mathbf{w}_{2}}\left(1+\frac{\hbar \varsigma^{\chi}}{\Gamma(\chi+1)}+\frac{\hbar^{2} \varsigma^{2 \chi}}{\Gamma(2 \chi+1)}+\frac{\hbar^{3} \varsigma^{3 \chi}}{\Gamma(3 \chi+1)}+\cdots\right)
\end{aligned}
$$

Case 2. Here, we surmise ABC fractional derivative operator coupled with the generalized integral transform and
Adomian decomposition method. Applying the generalized integral transform for Example 1,

$$
\frac{\psi^{\chi}(\mathfrak{g}) \mathbb{A}(\chi)}{\chi+(1-\chi) \psi^{\chi}(\mathfrak{g})} \mathscr{U}\left(\mathbf{w}_{1}, \mathfrak{g}\right)-\phi(\mathfrak{g}) \sum_{\kappa=0}^{m-1} \psi^{\chi-\kappa-1}(\mathfrak{g}) Q^{(\kappa)}(0)=\mathbb{J}\left[\mathcal{Q}_{\mathbf{w}_{1} \mathbf{w}_{1}}^{2}\left(\mathbf{w}_{1}, \mathbf{w}_{2}, \varsigma\right)+\mathbb{Q}_{\mathbf{w}_{2} \mathbf{w}_{2}}^{2}\left(\mathbf{w}_{1}, \mathbf{w}_{2}, \varsigma\right)+\hbar Q\left(\mathbf{w}_{1}, \mathbf{w}_{2}, \varsigma\right)\right]
$$

Taking into consideration the IC given in (36), we have

$$
\mathcal{U}\left(\mathbf{w}_{1}, \mathfrak{g}\right)=\frac{\psi(\mathfrak{g})}{\phi(\mathfrak{g})} \mathfrak{Q}\left(\mathbf{w}_{1}, \mathbf{w}_{2}, 0\right)+\frac{\chi+(1-\chi) \psi^{\chi}(\mathfrak{g})}{\psi^{\chi}(\mathfrak{g}) \mathbb{A}(\chi)} \mathbb{J}\left[\mathcal{Q}_{\mathbf{w}_{1} \mathbf{w}_{1}}^{2}\left(\mathbf{w}_{1}, \mathbf{w}_{2}, \varsigma\right)+Q_{\mathbf{w}_{2} \mathbf{w}_{2}}^{2}\left(\mathbf{w}_{1}, \mathbf{w}_{2}, \varsigma\right)+\hbar \mathbb{Q}\left(\mathbf{w}_{1}, \mathbf{w}_{2}, \varsigma\right)\right]
$$

Employing the inverse generalized integral transform, we obtain

$$
\mathcal{Q}\left(\mathbf{w}_{1}, \mathbf{w}_{2}, \varsigma\right)=\mathbb{J}^{-1}\left[\frac{\psi(\mathfrak{g})}{\phi(\mathfrak{G})} \mathcal{Q}\left(\mathbf{w}_{1}, \mathbf{w}_{2}, 0\right)+\frac{\chi+(1-\chi) \psi^{\chi}(\mathfrak{g})}{\psi^{\chi}(\mathfrak{g}) \mathbb{A}(\chi)} \mathbb{J}\left[\mathcal{Q}_{\mathbf{w}_{1} \mathbf{w}_{1}}^{2}\left(\mathbf{w}_{1}, \mathbf{w}_{2}, \varsigma\right)+\mathbb{Q}_{\mathbf{w}_{2} \mathbf{w}_{2}}^{2}\left(\mathbf{w}_{1}, \mathbf{w}_{2}, \varsigma\right)+\hbar Q\left(\mathbf{w}_{1}, \mathbf{w}_{2}, \varsigma\right)\right]\right]
$$
find

Thanks to the generalized decomposition method, we

$$
\begin{aligned}
\mathbb{Q}_{0}\left(\mathbf{w}_{1}, \mathbf{w}_{2}, \varsigma\right) & =\mathbb{J}^{-1}\left[\frac{\psi(\mathfrak{g})}{\phi(\mathfrak{g})} \mathscr{Q}\left(\mathbf{w}_{1}, \mathbf{w}_{2}, 0\right)\right]=\mathbb{J}^{-1}\left[\frac{\psi\left(s_{1}\right)}{\phi(\mathfrak{g})} \sqrt{\mathbf{w}_{1}+\mathbf{w}_{2}+\mathbf{w}_{1} \mathbf{w}_{2}}\right] \\
& =\sqrt{\mathbf{w}_{1}+\mathbf{w}_{2}+\mathbf{w}_{1} \mathbf{w}_{2}} .
\end{aligned}
$$

Here, we surmise that the unknown function Q $\left(\mathbf{w}_{1}, \mathbf{w}_{2}, \varsigma\right)$ can be written by an infinite series of the form

$$
\mathcal{Q}\left(\mathbf{w}_{1}, \mathbf{w}_{2}, \varsigma\right)=\sum_{\ell=0}^{\infty} Q_{\ell}\left(\mathbf{w}_{1}, \mathbf{w}_{2}, \varsigma\right)
$$

Also, the nonlinearity $\mathscr{F}_{j}(\mathcal{Q}), j=1,2$, can be decomposed by an infinite series of polynomials represented by

$$
\begin{aligned}
& \mathscr{F}_{1}(\mathcal{Q})=Q_{\mathbf{w}_{1} \mathbf{w}_{1}}^{2}=\sum_{\ell=0}^{\infty} \mathscr{A}_{\ell}, \\
& \mathscr{F}_{2}(\mathcal{Q})=Q_{\mathbf{w}_{2} \mathbf{w}_{2}}^{2}=\sum_{\ell=0}^{\infty} \mathscr{B}_{\ell},
\end{aligned}
$$

where $Q_{\ell}\left(\mathbf{w}_{1}, \mathbf{w}_{2}, \varsigma\right)$ will be evaluated recurrently and $\mathscr{A}_{\ell}$ and $\mathscr{B}_{\ell}$ are the so-called polynomials of $Q_{0}, Q_{1}, \ldots, Q_{\ell}$ defined in (44). 
For $\ell=0,1,2,3, \ldots$,

$$
\begin{aligned}
& \mathscr{Q}_{1}\left(\mathbf{w}_{1}, \mathbf{w}_{2}, \varsigma\right)=\mathbb{J}^{-1}\left[\frac{\chi+(1-\chi) \psi^{\chi}(\mathfrak{g})}{\psi^{\chi}(\mathfrak{g}) \mathbb{A}(\chi)} \mathbb{J}\left[\mathscr{A}_{0}+\mathscr{B}_{0}+\hbar \mathscr{Q}_{0}\right]\right] \\
& =\frac{\hbar \sqrt{\mathbf{w}_{1}+\mathbf{w}_{2}+\mathbf{w}_{1} \mathbf{w}_{2}}}{\mathbb{A}(\chi)}\left[\frac{\chi \varsigma^{\chi}}{\Gamma(\chi+1)}+(1-\chi)\right] \\
& \mathbb{Q}_{2}\left(\mathbf{w}_{1}, \mathbf{w}_{2}, \varsigma\right)=\mathbb{J}^{-1}\left[\frac{\chi+(1-\chi) \psi^{\chi}(\mathfrak{g})}{\psi^{\chi}(\mathfrak{g}) \mathbb{A}(\chi)} \mathbb{d}\left[\mathscr{A}_{1}+\mathscr{B}_{1}+\hbar \mathscr{Q}_{1}\right]\right] \\
& =\frac{\hbar^{2} \sqrt{\mathbf{w}_{1}+\mathbf{w}_{2}+\mathbf{w}_{1} \mathbf{w}_{2}}}{\mathbb{A}^{2}(\chi)}\left[\frac{\chi^{2} \varsigma^{2 \chi}}{\Gamma(2 \chi+1)}+2 \chi(1-\chi) \frac{\varsigma^{\chi}}{\Gamma(\chi+1)}+(1-\chi)^{2}\right] \text {, } \\
& Q_{3}\left(\mathbf{w}_{1}, \mathbf{w}_{2}, \varsigma\right)=\mathbb{J}^{-1}\left[\frac{\chi+(1-\chi) \psi^{\chi}(\mathfrak{g})}{\psi^{\chi}(\mathfrak{G}) \mathbb{A}(\chi)} \mathbb{I}\left[\mathscr{A}_{2}+\mathscr{B}_{2}+\hbar Q_{2}\right]\right] \\
& =\frac{\hbar^{3} \sqrt{\mathbf{w}_{1}+\mathbf{w}_{2}+\mathbf{w}_{1} \mathbf{w}_{2}}}{\mathbb{A}^{3}(\chi)}\left[\frac{\chi^{3} \varsigma^{3 \chi}}{\Gamma(3 \chi+1)}+3 \chi^{2}(1-\chi) \frac{\varsigma^{2 \chi}}{\Gamma(2 \chi+1)}+3 \chi(1-\chi)^{2} \frac{\varsigma^{\chi}}{\Gamma(\chi+1)}+(1-\chi)^{3}\right] \text {, } \\
& \vdots
\end{aligned}
$$
follows:

The approximate solution for Example 1 is expressed as

$$
\begin{aligned}
Q\left(\mathbf{w}_{1}, \mathbf{w}_{2}, \varsigma\right)= & \Phi_{0}\left(\mathbf{w}_{1}, \mathbf{w}_{2}, \varsigma\right)+\Phi_{1}\left(\mathbf{w}_{1}, \mathbf{w}_{2}, \varsigma\right)+\Phi_{2}\left(\mathbf{w}_{1}, \mathbf{w}_{2}, \varsigma\right)+\Phi_{3}\left(\mathbf{w}_{1}, \mathbf{w}_{2}, \varsigma\right)+\cdots \\
= & \sqrt{\mathbf{w}_{1}+\mathbf{w}_{2}+\mathbf{w}_{1} \mathbf{w}_{2}}\left(1+\frac{\hbar}{\mathbb{A}(\chi)}\left(\frac{\varsigma^{\chi}}{\Gamma(\chi+1)}+(1-\chi)\right)\right. \\
& +\frac{\hbar^{2}}{\mathbb{A}^{2}(\chi)}\left(\frac{\chi^{2} \varsigma^{2 \chi}}{\Gamma(2 \chi+1)}+2 \chi(1-\chi) \frac{\varsigma^{\chi}}{\Gamma(\chi+1)}+(1-\chi)^{2}\right) \\
& \left.+\frac{\hbar^{3}}{\mathbb{A}^{3}(\chi)}\left(\frac{\chi^{3} \varsigma^{3 \chi}}{\Gamma(3 \chi+1)}+3 \chi^{2}(1-\chi) \frac{\varsigma^{2 \chi}}{\Gamma(2 \chi+1)}+3 \chi(1-\chi)^{2} \frac{\varsigma^{\chi}}{\Gamma(\chi+1)}+(1-\chi)^{3}\right)+\cdots\right)
\end{aligned}
$$

For $\chi=1$, we obtained the exact solution of Example 1 as

$$
Q\left(\mathbf{w}_{1}, \mathbf{w}_{2}, \varsigma\right)=\sqrt{\mathbf{w}_{1}+\mathbf{w}_{2}+\mathbf{w}_{1} \mathbf{w}_{2}} \exp (\hbar \varsigma) .
$$

The analytical approximate solutions including certain random initialization produced by the proposed methodology are shown in Table 1. The VIMHP is employed to perform the comparative analysis, which forecasts the precision of the proposed methodology based on its lower error. The findings in this study are tremendously helpful in comprehending the internal components of natural disasters. We will describe the scientific clarification of the solutions for the BP model in this paragraph of the article. The exact and numerical solution for $\alpha=1, r_{1}=0, \varsigma=0.01$, and $\chi=1$ is shown in Figure 1.
Furthermore, the absolute error for the aforementioned assumptions is depicted in Figure 2(a). Figure 2(b) demonstrates the behavior of the findings in three-dimensional simulation for different fractional orders by employing the Caputo fractional derivative operator.

Finally, Figure 3 represents the two-dimensional behavior of exact, approximate by Caputo, and approximate solutions derived by $\mathrm{ABC}$ fractional operators with varying fractional orders. These solutions have a distinctive characteristic that allows them to interact with other solutions derived by [23]. The proposed findings have particle-like geometries in their solutions. The synthesized trajectory is either a success or a descent from one asymptotic state to the next. The accuracy of the proposed method can be enhanced by increasing the recursive terms. 
TABLE 1: Exact $\mathbb{Q}_{E}$ and approximate solutions $\mathbb{Q}_{\mathrm{CFD}}$ and $\mathbb{Q}_{\mathrm{ABC}}$ of $\mathbb{Q}\left(\mathbf{w}_{1}, \mathbf{w}_{2}, \varsigma\right)$ of Example 1 having absolute errors $E_{1}=\left\|\mathbb{Q}_{E}-\mathbb{Q}_{\mathrm{CFD}}\right\|$ and $E_{2}=\left\|Q_{E}-Q_{\mathrm{ABC}}\right\|$ when $\chi=1, \varsigma=0.2, r_{1}=0$, and $\hbar=1 / 2$ for different values of $\mathbf{w}_{1}$ and $\mathbf{w}_{2}$.

\begin{tabular}{|c|c|c|c|c|c|c|}
\hline$\left(\mathbf{w}_{1}, \mathbf{w}_{2}\right)$ & $\mathscr{Q}_{E}$ sol. & $\mathbb{Q}_{\mathrm{CFD}}$ sol. & $\mathcal{Q}_{\mathrm{ABC}}$ sol. & VIMHP sol. [23] & Error $=E_{1}$ & Error $=E_{2}$ \\
\hline$(-10,10)$ & 11.051709 & 11.051666 & 11.051666 & 11.051344 & $4.2510 e-5$ & $4.2510 e-5$ \\
\hline$(-8,8)$ & 8.841367 & 8.841333 & 8.841333 & 8.841600 & $3.4008 e-5$ & $3.4008 e-5$ \\
\hline$(-6,6)$ & 6.631025 & 6.631000 & 6.631000 & 6.631555 & $2.5506 e-5$ & $2.5506 e-5$ \\
\hline$(-4,4)$ & 6.631025 & 6.631000 & 6.631000 & 6.667890 & $2.5506 e-5$ & $2.5506 e-5$ \\
\hline$(-2,2)$ & 2.210341 & 2.210333 & 2.210333 & 2.226699 & $8.5020 e-6$ & $8.5020 e-6$ \\
\hline$(0,0)$ & 0.000000 & 0.000000 & 0.000000 & 1.008975 & 0.000000 & 0.000000 \\
\hline$(2,2)$ & 3.125800 & 3.125800 & 3.125800 & 3.998500 & $1.2024 e-5$ & $1.2024 e-5$ \\
\hline$(4,4)$ & 5.414100 & 5.414100 & 5.414100 & 6.889200 & $2.0826 e-5$ & $2.0826 e-5$ \\
\hline$(6,6)$ & 7.657100 & 7.657100 & 7.657100 & 8.100650 & $2.9453 e-5$ & $2.9453 e-5$ \\
\hline$(8,8)$ & 9.885100 & 9.885100 & 9.885100 & 9.987890 & $3.8023 e-5$ & $3.8023 e-5$ \\
\hline$(10,10)$ & 1.210600 & 1.210600 & 1.210600 & 1.9823 & $4.6567 e-5$ & $4.6567 e-5$ \\
\hline
\end{tabular}

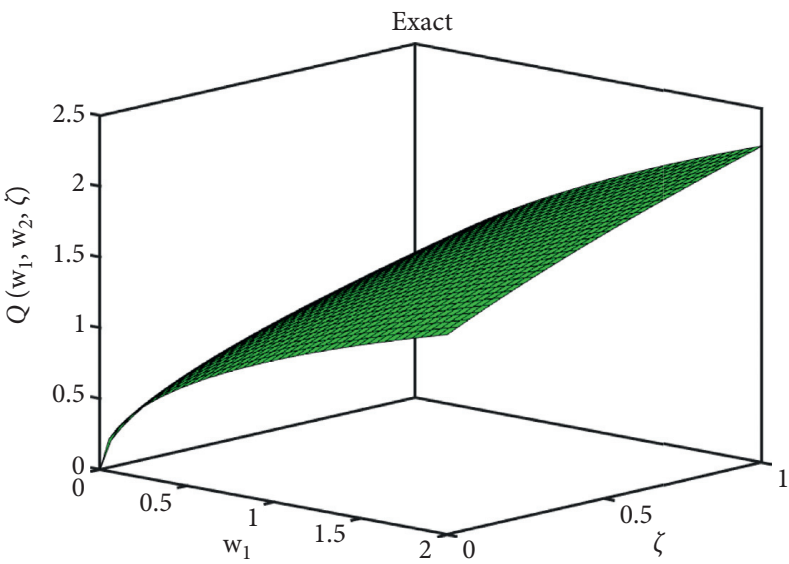

(a)

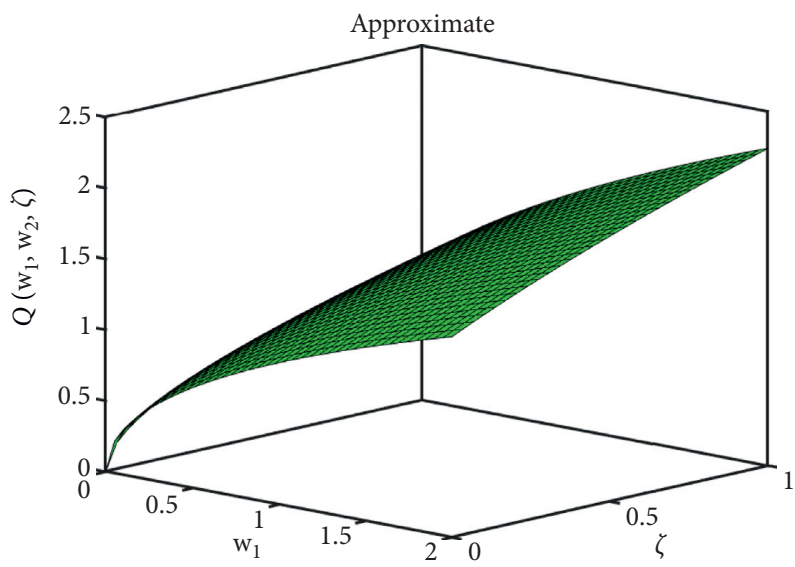

(b)

FIgURE 1: Three-dimensional illustration of the exact and approximate solution of Example 1 when $\alpha=1, r_{1}=0, \varsigma=0.01$, and $\chi=1$.

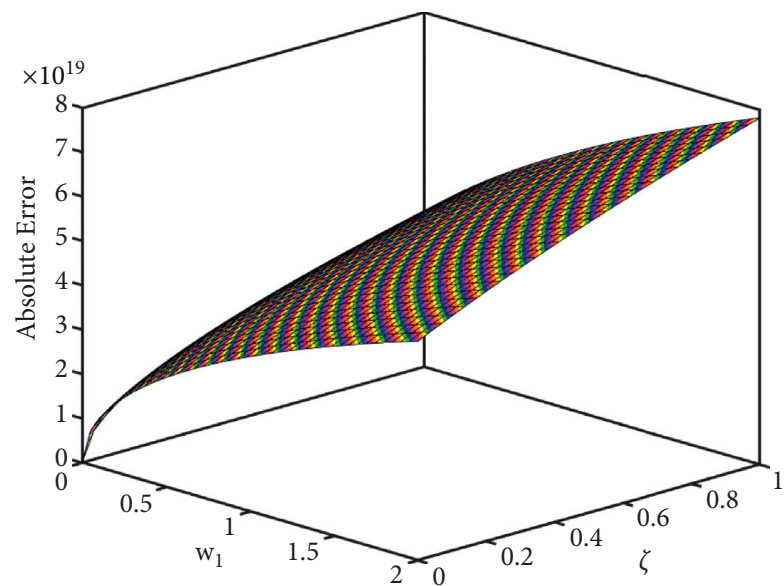

(a)

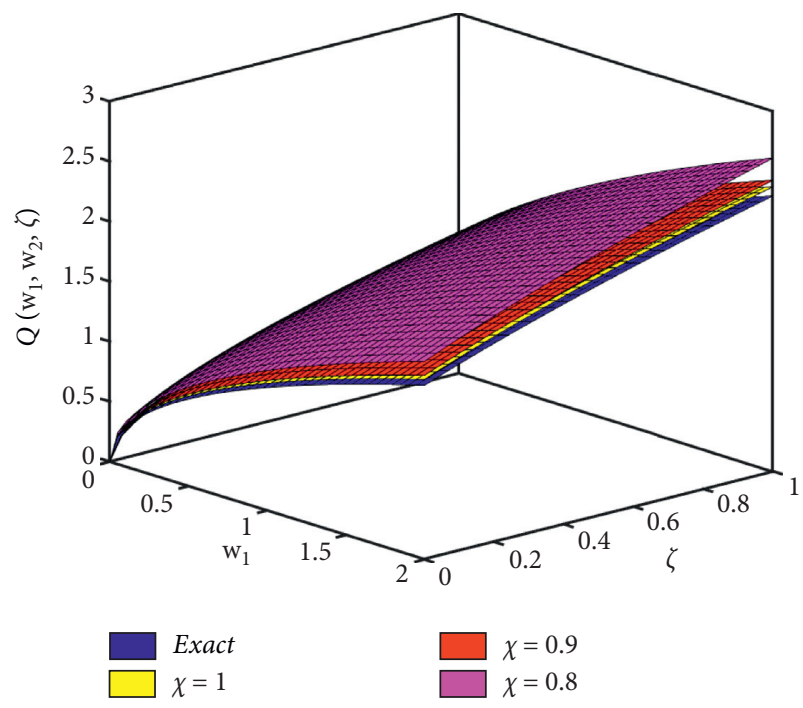

(b)

Figure 2: Three-dimensional illustration of the absolute error and multiple surface of various fractional orders for Example 1 when $\alpha=1, r_{1}=0$, and $\varsigma=0.01$. 


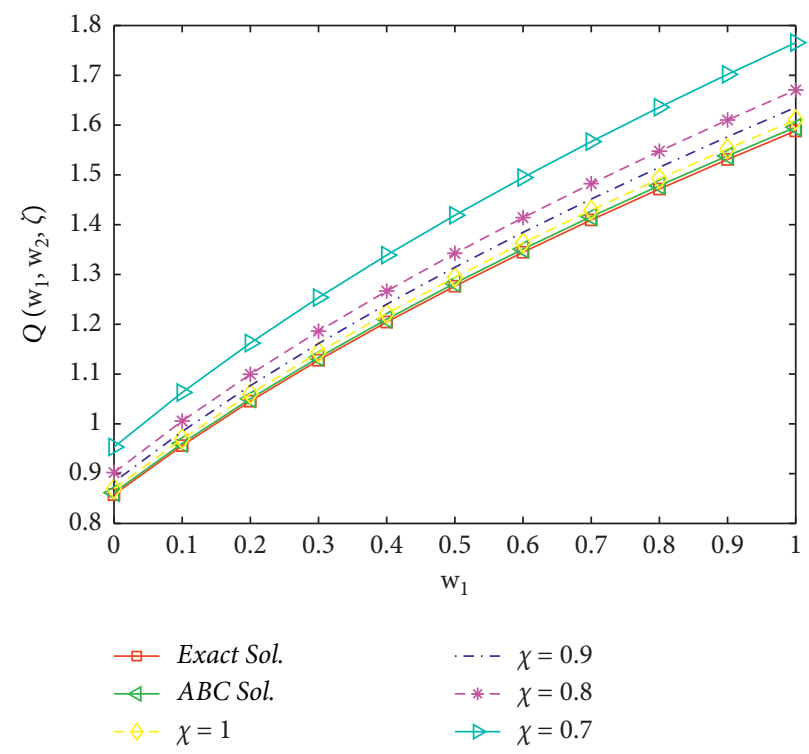

FIgURE 3: Two-dimensional illustration of Example 1 for various fractional orders when $\alpha=1, r_{1}=0$, and $\varsigma=0.01$.

Remark 3. Example 1 leads to the conclusion that

(i) If we replace the IC $Q\left(\mathbf{w}_{1}, \mathbf{w}_{2}, \varsigma\right)=$ $\sqrt{\alpha_{1} \mathbf{w}_{1}+\beta_{1} \mathbf{w}_{2}+\gamma_{1} \mathbf{w}_{1} \mathbf{w}_{2}}$ in Example 1, then the approximate solution can be achieved as

$$
\mathcal{Q}\left(\mathbf{w}_{1}, \mathbf{w}_{2}, \varsigma\right)=\sqrt{\alpha_{1} \mathbf{w}_{1}+\beta_{1} \mathbf{w}_{2}+\gamma_{1} \mathbf{w}_{1} \mathbf{w}_{2}}\left(1+\frac{\hbar \varsigma^{\chi}}{\Gamma(\chi+1)}+\frac{\hbar^{2} \varsigma^{2 \chi}}{\Gamma(2 \chi+1)}+\frac{\hbar^{3} \varsigma^{3 \chi}}{\Gamma(3 \chi+1)}+\cdots\right)
$$

(ii) The closed form solution in the stated case will be $Q\left(\mathbf{w}_{1}, \mathbf{w}_{2}, \varsigma\right)=\sqrt{\alpha_{1} \mathbf{w}_{1}+\beta_{1} \mathbf{w}_{2}+\gamma_{1} \mathbf{w}_{1} \mathbf{w}_{2}} \exp (\hbar \varsigma)$, where $\alpha_{1}$ and $\beta_{1}$ are the real constants.

(iii) Letting $\alpha_{1}=\beta_{1}=0$ and $\gamma_{1}=1$ along with $\hbar=1 / 2$, we get the result proposed independently by Roul [53] and Shakeri and Dehgan [54], respectively.
Example 2. Assume the time-fractional BPM (4) having $\alpha=1, r_{1}=0$, and $\hbar=1$; then,

$$
\mathbf{D}_{\varsigma}^{\alpha} Q\left(\mathbf{w}_{1}, \mathbf{w}_{2}, \varsigma\right)=Q_{\mathbf{w}_{1} \mathbf{w}_{1}}^{2}\left(\mathbf{w}_{1}, \mathbf{w}_{2}, \varsigma\right)+Q_{\mathbf{w}_{2} \mathbf{w}_{2}}^{2}\left(\mathbf{w}_{1}, \mathbf{w}_{2}, \varsigma\right)+Q\left(\mathbf{w}_{1}, \mathbf{w}_{2}, \varsigma\right),
$$

with IC

$$
\widehat{Q}_{0}\left(\mathbf{w}_{1}, \mathbf{w}_{2}, 0\right)=\sqrt{\sin \left(\theta \mathbf{w}_{1}\right) \cosh \left(\theta \mathbf{w}_{2}\right)}, \quad \theta \in \mathbb{R} .
$$

Case 1. First, we apply the Caputo fractional derivative operator coupled with the generalized integral transform and Adomian decomposition method. Applying the generalized integral transform for Example 2,

Proof. . Foremost, we provide the solution of (57) in two general cases.

$$
\psi^{\chi}(\mathfrak{g}) \mathcal{U}\left(\mathbf{w}_{1}, \mathfrak{g}\right)-\phi(\mathfrak{g}) \sum_{\kappa=0}^{m-1} \psi^{\chi-\kappa-1}(\mathfrak{g}) \mathscr{Q}^{(\kappa)}(0)=\mathbb{J}\left[\mathcal{Q}_{\mathbf{w}_{1} \mathbf{w}_{1}}^{2}\left(\mathbf{w}_{1}, \mathbf{w}_{2}, \varsigma\right)+\mathbb{Q}_{\mathbf{w}_{2} \mathbf{w}_{2}}^{2}\left(\mathbf{w}_{1}, \mathbf{w}_{2}, \varsigma\right)+\mathscr{Q}\left(\mathbf{w}_{1}, \mathbf{w}_{2}, \varsigma\right)\right]
$$

Taking into consideration the IC given in (58), we have 


$$
\mathcal{U}\left(\mathbf{w}_{1}, \mathfrak{G}\right)=\frac{\psi(\mathfrak{g})}{\phi(\mathfrak{g})} \mathscr{Q}\left(\mathbf{w}_{1}, \mathbf{w}_{2}, 0\right)+\frac{1}{\psi^{X}(\mathfrak{g})} \mathbb{J}\left[\widehat{Q}_{\mathbf{w}_{1} \mathbf{w}_{1}}^{2}\left(\mathbf{w}_{1}, \mathbf{w}_{2}, \varsigma\right)+\mathbb{Q}_{\mathbf{w}_{2} \mathbf{w}_{2}}^{2}\left(\mathbf{w}_{1}, \mathbf{w}_{2}, \varsigma\right)+\mathscr{Q}\left(\mathbf{w}_{1}, \mathbf{w}_{2}, \varsigma\right)\right] .
$$

Employing the inverse generalized integral transform, we obtain

$$
\mathscr{Q}\left(\mathbf{w}_{1}, \mathbf{w}_{2}, \varsigma\right)=\mathbb{J}^{-1}\left[\frac{\psi(\mathfrak{g})}{\phi(\mathfrak{g})} \mathscr{Q}\left(\mathbf{w}_{1}, \mathbf{w}_{2}, 0\right)+\frac{1}{\psi^{\chi}(\mathfrak{g})} \mathbb{J}\left[\mathscr{Q}_{\mathbf{w}_{1} \mathbf{w}_{1}}^{2}\left(\mathbf{w}_{1}, \mathbf{w}_{2}, \varsigma\right)+\mathbb{Q}_{\mathbf{w}_{2} \mathbf{w}_{2}}^{2}\left(\mathbf{w}_{1}, \mathbf{w}_{2}, \varsigma\right)+\mathscr{Q}\left(\mathbf{w}_{1}, \mathbf{w}_{2}, \varsigma\right)\right]\right]
$$

Thanks to the generalized decomposition method, we find

$$
\begin{aligned}
\mathbb{Q}_{0}\left(\mathbf{w}_{1}, \mathbf{w}_{2}, \varsigma\right) & =\mathbb{J}^{-1}\left[\frac{\psi(\mathfrak{g})}{\phi(\mathfrak{g})} \mathscr{Q}\left(\mathbf{w}_{1}, \mathbf{w}_{2}, 0\right)\right]=\mathbb{J}^{-1}\left[\frac{\psi(\mathfrak{g})}{\phi(\mathfrak{g})} \sqrt{\sin \left(\theta \mathbf{w}_{1}\right) \cosh \left(\theta \mathbf{w}_{2}\right)}\right] \\
& =\sqrt{\sin \left(\theta \mathbf{w}_{1}\right) \cosh \left(\theta \mathbf{w}_{2}\right)} .
\end{aligned}
$$

Here, we surmise that the unknown function $Q\left(\mathbf{w}_{1}, \mathbf{w}_{2}, \varsigma\right)$ can be written by an infinite series of the form

$$
Q\left(\mathbf{w}_{1}, \mathbf{w}_{2}, \varsigma\right)=\sum_{\ell=0}^{\infty} Q_{\ell}\left(\mathbf{w}_{1}, \mathbf{w}_{2}, \varsigma\right) .
$$

Also, the nonlinearity $\mathscr{F}_{j}(\mathbb{Q}), j=1,2$, can be decomposed by an infinite series of polynomials represented by $\mathscr{F}_{1}(Q)=Q_{\mathbf{w}_{1} \mathbf{w}_{1}}^{2}=\sum_{\ell=0}^{\infty} \mathscr{A}_{\ell}$ and $\mathscr{F}_{2}(\mathbb{Q})=\mathbb{Q}_{\mathbf{w}_{2} \mathbf{w}_{2}}^{2}=\sum_{\ell=0}^{\infty} \mathscr{B}_{\ell}$ defined in (44).

$$
\sum_{\ell=0}^{\infty} Q_{\ell+1}\left(\mathbf{w}_{1}, \mathbf{w}_{2}, \varsigma\right)=\mathbb{J}^{-1}\left[\frac{1}{\psi^{X}(\mathfrak{G})} \sqrt{ }\left[\sum_{\ell=0}^{\infty}(\mathscr{A})_{\ell}+\sum_{\ell=0}^{\infty}(\mathscr{B})_{\ell}+\sum_{\ell=0}^{\infty}(\mathscr{Q})_{\ell}\right]\right], \quad \ell=0,1,2, \ldots
$$

For $\ell=0,1,2,3, \ldots$,

$$
\begin{aligned}
\mathbb{Q}_{1}\left(\mathbf{w}_{1}, \mathbf{w}_{2}, \varsigma\right) & =\mathbb{J}^{-1}\left[\frac{1}{\psi^{\chi}(\mathfrak{g})} \mathbb{D}\left[\mathscr{A}_{0}+\mathscr{B}_{0}+\hbar \mathscr{Q}_{0}\right]\right] \\
& =\sqrt{\sin \left(\theta \mathbf{w}_{1}\right) \cosh \left(\theta \mathbf{w}_{2}\right)} \frac{\varsigma^{\chi}}{\Gamma(\chi+1)}, \\
\mathbb{Q}_{2}\left(\mathbf{w}_{1}, \mathbf{w}_{2}, \varsigma\right) & =\mathbb{J}^{-1}\left[\frac{1}{\psi^{\chi}(\mathfrak{g})} \mathbb{D}\left[\mathscr{A}_{1}+\mathscr{B}_{1}+\hbar \mathscr{Q}_{1}\right]\right] \\
& =\sqrt{\sin \left(\theta \mathbf{w}_{1}\right) \cosh \left(\theta \mathbf{w}_{2}\right)} \frac{\varsigma^{2 \chi}}{\Gamma(2 \chi+1)}, \\
\mathbb{Q}_{3}\left(\mathbf{w}_{1}, \mathbf{w}_{2}, \varsigma\right) & =\mathbb{J}^{-1}\left[\frac{1}{\psi^{\chi}(\mathfrak{g})} \mathbb{D}\left[\mathscr{A}_{2}+\mathscr{B}_{2}+\hbar \mathscr{Q}_{2}\right]\right] \\
& =\sqrt{\sin \left(\theta \mathbf{w}_{1}\right) \cosh \left(\theta \mathbf{w}_{2}\right)} \frac{\varsigma^{3 \chi}}{\Gamma(3 \chi+1)},
\end{aligned}
$$


The approximate solution for Example 2 is expressed as follows:

$$
\begin{aligned}
\mathscr{Q}\left(\mathbf{w}_{1}, \mathbf{w}_{2}, \varsigma\right) & =\Phi_{0}\left(\mathbf{w}_{1}, \mathbf{w}_{2}, \varsigma\right)+\Phi_{1}\left(\mathbf{w}_{1}, \mathbf{w}_{2}, \varsigma\right)+\Phi_{2}\left(\mathbf{w}_{1}, \mathbf{w}_{2}, \varsigma\right)+\Phi_{3}\left(\mathbf{w}_{1}, \mathbf{w}_{2}, \varsigma\right)+\cdots \\
& =\sqrt{\sin \left(\theta \mathbf{w}_{1}\right) \cosh \left(\theta \mathbf{w}_{2}\right)}\left(1+\frac{\varsigma^{\chi}}{\Gamma(\chi+1)}+\frac{\varsigma^{2 \chi}}{\Gamma(2 \chi+1)}+\frac{\varsigma^{3 \chi}}{\Gamma(3 \chi+1)}+\cdots\right) .
\end{aligned}
$$

Case 2. Here, we surmise $\mathrm{ABC}$ fractional derivative operator coupled with the generalized integral transform and
Adomian decomposition method. Applying the generalized integral transform for Example 2,

$$
\frac{\psi^{\chi}(\mathfrak{g}) \mathbb{A}(\chi)}{\chi+(1-\chi) \psi^{\chi}(\mathfrak{g})} \mathscr{U}\left(\mathbf{w}_{1}, \mathfrak{g}\right)-\phi(\mathfrak{g}) \sum_{\kappa=0}^{m-1} \psi^{\chi-\kappa-1}(\mathfrak{G}) \mathbb{Q}^{(\kappa)}(0)=\mathbb{J}\left[\mathbb{Q}_{\mathbf{w}_{1} \mathbf{w}_{1}}^{2}\left(\mathbf{w}_{1}, \mathbf{w}_{2}, \varsigma\right)+\mathbb{Q}_{\mathbf{w}_{2} \mathbf{w}_{2}}^{2}\left(\mathbf{w}_{1}, \mathbf{w}_{2}, \varsigma\right)+\mathscr{Q}\left(\mathbf{w}_{1}, \mathbf{w}_{2}, \varsigma\right)\right] .
$$

Taking into consideration the IC given in (58), we have

$$
\mathscr{U}\left(\mathbf{w}_{1}, \mathfrak{g}\right)=\frac{\psi(\mathfrak{g})}{\phi(\mathfrak{g})} \mathscr{Q}\left(\mathbf{w}_{1}, \mathbf{w}_{2}, 0\right)+\frac{\chi+(1-\chi) \psi^{\chi}(\mathfrak{g})}{\psi^{\chi}(\mathfrak{g}) \mathbb{A}(\chi)} \mathbb{J}\left[\mathcal{Q}_{\mathbf{w}_{1} \mathbf{w}_{1}}^{2}\left(\mathbf{w}_{1}, \mathbf{w}_{2}, \varsigma\right)+\mathbb{Q}_{\mathbf{w}_{2} \mathbf{w}_{2}}^{2}\left(\mathbf{w}_{1}, \mathbf{w}_{2}, \varsigma\right)+\mathscr{Q}\left(\mathbf{w}_{1}, \mathbf{w}_{2}, \varsigma\right)\right] .
$$

Employing the inverse generalized integral transform, we obtain

$$
\mathscr{Q}\left(\mathbf{w}_{1}, \mathbf{w}_{2}, \varsigma\right)=\mathbb{J}^{-1}\left[\frac{\psi(\mathfrak{g})}{\phi(\mathfrak{g})} \mathscr{Q}\left(\mathbf{w}_{1}, \mathbf{w}_{2}, 0\right)+\frac{\chi+(1-\chi) \psi^{\chi}(\mathfrak{g})}{\psi^{\chi}(\mathfrak{g}) \mathbb{A}(\chi)} \mathbb{J}\left[\mathscr{Q}_{\mathbf{w}_{1} \mathbf{w}_{1}}^{2}\left(\mathbf{w}_{1}, \mathbf{w}_{2}, \varsigma\right)+\mathscr{Q}_{\mathbf{w}_{2} \mathbf{w}_{2}}^{2}\left(\mathbf{w}_{1}, \mathbf{w}_{2}, \varsigma\right)+\mathscr{Q}\left(\mathbf{w}_{1}, \mathbf{w}_{2}, \varsigma\right)\right]\right]
$$
find

Thanks to the generalized decomposition method, we

$$
\begin{aligned}
& \mathbb{Q}_{0}\left(\mathbf{w}_{1}, \mathbf{w}_{2}, \varsigma\right)=\mathbb{J}^{-1}\left[\frac{\psi(\mathfrak{g})}{\phi(\mathfrak{I})} \mathscr{Q}\left(\mathbf{w}_{1}, \mathbf{w}_{2}, 0\right)\right]=\mathbb{J}^{-1}\left[\frac{\psi(\mathfrak{g})}{\phi(\mathfrak{I})} \sqrt{\sin \left(\theta \mathbf{w}_{1}\right) \cosh \left(\theta \mathbf{w}_{2}\right)}\right] \\
& =\sqrt{\sin \left(\theta \mathbf{w}_{1}\right) \cosh \left(\theta \mathbf{w}_{2}\right)} .
\end{aligned}
$$

Here, we surmise that the unknown function $\mathbb{Q}\left(\mathbf{w}_{1}, \mathbf{w}_{2}, \varsigma\right)$ can be written by an infinite series of the form

$$
Q\left(\mathbf{w}_{1}, \mathbf{w}_{2}, \varsigma\right)=\sum_{\ell=0}^{\infty} Q_{\ell}\left(\mathbf{w}_{1}, \mathbf{w}_{2}, \varsigma\right) .
$$

Also, the nonlinearity $\mathscr{F}_{j}(\mathscr{Q}), j=1,2$, can be decomposed by an infinite series of polynomials represented by

$$
\begin{aligned}
& \mathscr{F}_{1}(Q)=Q_{\mathbf{w}_{1} \mathbf{w}_{1}}^{2}=\sum_{\ell=0}^{\infty} \mathscr{A}_{\ell}, \\
& \mathscr{F}_{2}(Q)=Q_{\mathbf{w}_{2} \mathbf{w}_{2}}^{2}=\sum_{\ell=0}^{\infty} \mathscr{B}_{\ell},
\end{aligned}
$$

where $\mathbb{Q}_{\ell}\left(\mathbf{w}_{1}, \mathbf{w}_{2}, \varsigma\right)$ will be evaluated recurrently and $\mathscr{A}_{\ell}$ and $\mathscr{B}_{\ell}$ are the so-called polynomials of $\mathbb{Q}_{0}, \mathbb{Q}_{1}, \ldots, \mathbb{Q}_{\ell}$ defined in (44). 
For $\ell=0,1,2,3, \ldots$,

$$
\begin{aligned}
& \mathscr{Q}_{1}\left(\mathbf{w}_{1}, \mathbf{w}_{2}, \varsigma\right)=\mathbb{J}^{-1}\left[\frac{\chi+(1-\chi) \psi^{\chi}(\mathfrak{g})}{\psi^{\chi}(\mathfrak{g}) \mathbb{A}(\chi)} \mathbb{J}\left[\mathscr{A}_{0}+\mathscr{B}_{0}+\mathscr{Q}_{0}\right]\right] \\
& =\frac{\sqrt{\sin \left(\theta \mathbf{w}_{1}\right) \cosh \left(\theta \mathbf{w}_{2}\right)}}{\mathbb{A}(\chi)}\left[\frac{\chi \varsigma^{\chi}}{\Gamma(\chi+1)}+(1-\chi)\right] \\
& \mathbb{Q}_{2}\left(\mathbf{w}_{1}, \mathbf{w}_{2}, \varsigma\right)=\mathbb{J}^{-1}\left[\frac{\chi+(1-\chi) \psi^{\chi}(\mathfrak{g})}{\psi^{\chi}(\mathfrak{g}) \mathbb{A}(\chi)} \mathbb{J}\left[\mathscr{A}_{1}+\mathscr{B}_{1}+\mathscr{Q}_{1}\right]\right] \\
& =\frac{\sqrt{\sin \left(\theta \mathbf{w}_{1}\right) \cosh \left(\theta \mathbf{w}_{2}\right)}}{\mathbb{A}^{2}(\chi)}\left[\frac{\chi^{2} \varsigma^{2 \chi}}{\Gamma(2 \chi+1)}+2 \chi(1-\chi) \frac{\varsigma^{\chi}}{\Gamma(\chi+1)}+(1-\chi)^{2}\right], \\
& \mathbb{Q}_{3}\left(\mathbf{w}_{1}, \mathbf{w}_{2}, \varsigma\right)=\mathbb{J}^{-1}\left[\frac{\chi+(1-\chi) \psi^{\chi}(\mathfrak{g})}{\psi^{\chi}(\mathfrak{g}) \mathbb{A}(\chi)} \mathbb{J}\left[\mathscr{A}_{2}+\mathscr{B}_{2}+\mathscr{Q}_{2}\right]\right] \\
& =\frac{\sqrt{\sin \left(\theta \mathbf{w}_{1}\right) \cosh \left(\theta \mathbf{w}_{2}\right)}}{\mathbb{A}^{3}(\chi)}\left[\frac{\chi^{3} \varsigma^{3 \chi}}{\Gamma(3 \chi+1)}+3 \chi^{2}(1-\chi) \frac{\varsigma^{2 \chi}}{\Gamma(2 \chi+1)}+3 \chi(1-\chi)^{2} \frac{\varsigma^{\chi}}{\Gamma(\chi+1)}+(1-\chi)^{3}\right], \\
& \vdots
\end{aligned}
$$

The approximate solution for Example 2 is expressed as follows:

$$
\begin{aligned}
Q\left(\mathbf{w}_{1}, \mathbf{w}_{2}, \varsigma\right)= & \Phi_{0}\left(\mathbf{w}_{1}, \mathbf{w}_{2}, \varsigma\right)+\Phi_{1}\left(\mathbf{w}_{1}, \mathbf{w}_{2}, \varsigma\right)+\Phi_{2}\left(\mathbf{w}_{1}, \mathbf{w}_{2}, \varsigma\right)+\Phi_{3}\left(\mathbf{w}_{1}, \mathbf{w}_{2}, \varsigma\right)+\cdots \\
= & \sqrt{\sin \left(\theta \mathbf{w}_{1}\right) \cosh \left(\theta \mathbf{w}_{2}\right)}\left(1+\frac{1}{\mathbb{A}(\chi)}\left(\frac{\varsigma^{\chi}}{\Gamma(\chi+1)}+(1-\chi)\right)\right. \\
& +\frac{1}{\mathbb{A}^{2}(\chi)}\left(\frac{\chi^{2} \varsigma^{2 \chi}}{\Gamma(2 \chi+1)}+2 \chi(1-\chi) \frac{\varsigma^{\chi}}{\Gamma(\chi+1)}+(1-\chi)^{2}\right) \\
& \left.+\frac{1}{\mathbb{A}^{3}(\chi)}\left(\frac{\chi^{3} \varsigma^{3 \chi}}{\Gamma(3 \chi+1)}+3 \chi^{2}(1-\chi) \frac{\varsigma^{2 \chi}}{\Gamma(2 \chi+1)}+3 \chi(1-\chi)^{2} \frac{\varsigma^{\chi}}{\Gamma(\chi+1)}+(1-\chi)^{3}\right)+\cdots\right)
\end{aligned}
$$

For $\chi=1$, we obtained the exact solution of Example 2 as

$$
Q\left(\mathbf{w}_{1}, \mathbf{w}_{2}, \varsigma\right)=\sqrt{\sin \left(\theta \mathbf{w}_{1}\right) \cosh \left(\theta \mathbf{w}_{2}\right)} \exp (\varsigma) .
$$

The analytical approximate solutions including certain random initialization produced by the proposed methodology are shown in Table 2. The VIMHP is employed to perform the comparative analysis, which forecasts the precision of the proposed methodology based on its lower error. The findings in this study are tremendously helpful in comprehending the internal components of natural disasters. We will describe the scientific clarification of the solutions for the BP model in this paragraph of the article.
The exact and numerical solution for $\alpha=1, r_{1}=0, \varsigma=0.01$, and $\hbar=\chi=1$ is shown in Figure 4.

Furthermore, the absolute error for the aforementioned assumptions is depicted in Figure 5(a). Figure 5(b) demonstrates the behavior of the findings in three-dimensional simulation for different fractional orders by employing the Caputo fractional derivative operator.

Finally, Figure 6 represents the two-dimensional behavior of exact, approximate by Caputo, and approximate solutions derived by $\mathrm{ABC}$ fractional operators with varying fractional orders. These solutions have a distinctive characteristic that allows them to interact with other solutions derived by [23]. The proposed findings have particle-like 
geometries in their solutions. The synthesized trajectory is either a success or a descent from one asymptotic state to the next. The accuracy of the proposed method can be enhanced by increasing the recursive terms.
Example 3 (Verhulst law [19]). Assume the time-fractional BPM (4) having $\alpha=\beta=1$; then,

$$
\mathbf{D}_{\varsigma}^{\alpha} Q\left(\mathbf{w}_{1}, \mathbf{w}_{2}, \varsigma\right)=Q_{\mathbf{w}_{1} \mathbf{w}_{1}}^{2}\left(\mathbf{w}_{1}, \mathbf{w}_{2}, \varsigma\right)+\mathbb{Q}_{\mathbf{w}_{2} \mathbf{w}_{2}}^{2}\left(\mathbf{w}_{1}, \mathbf{w}_{2}, \varsigma\right)+\hbar Q\left(\mathbf{w}_{1}, \mathbf{w}_{2}, \varsigma\right)\left(1-r_{1} Q\left(\mathbf{w}_{1}, \mathbf{w}_{2}, \varsigma\right)\right)
$$

with IC

$$
Q_{0}\left(\mathbf{w}_{1}, \mathbf{w}_{2}, 0\right)=\exp \left(\sqrt{\frac{\hbar r_{1}}{8}}\left(\mathbf{w}_{1}+\mathbf{w}_{2}\right)\right)
$$

Proof. Foremost, we provide the solution of (76) in two general cases.
Case 1. First, we apply the Caputo fractional derivative operator coupled with the generalized integral transform and Adomian decomposition method. Applying the generalized integral transform for Example 3,

$$
\begin{aligned}
\psi^{\chi}(\mathfrak{g}) \mathcal{U}\left(\mathbf{w}_{1}, \mathfrak{g}\right)-\phi(\mathfrak{g}) \sum_{\kappa=0}^{m-1} \psi^{\chi-\kappa-1}(\mathfrak{g}) Q^{(\kappa)}(0)= & \mathbb{I}\left[\mathcal{Q}_{\mathbf{w}_{1} \mathbf{w}_{1}}^{2}\left(\mathbf{w}_{1}, \mathbf{w}_{2}, \varsigma\right)+Q_{\mathbf{w}_{2} \mathbf{w}_{2}}^{2}\left(\mathbf{w}_{1}, \mathbf{w}_{2}, \varsigma\right)\right. \\
& \left.+\hbar \mathscr{Q}\left(\mathbf{w}_{1}, \mathbf{w}_{2}, \varsigma\right)\left(1-r_{1} \mathbb{Q}\left(\mathbf{w}_{1}, \mathbf{w}_{2}, \varsigma\right)\right)\right] .
\end{aligned}
$$

Taking into consideration the IC given in (77), we have

$$
\mathcal{U}\left(\mathbf{w}_{1}, \mathfrak{g}\right)=\frac{\psi(\mathfrak{g})}{\phi(\mathfrak{g})} \mathbb{Q}\left(\mathbf{w}_{1}, \mathbf{w}_{2}, 0\right)+\frac{1}{\psi^{\chi}(\mathfrak{g})} \mathbb{J}\left[\mathcal{Q}_{\mathbf{w}_{1} \mathbf{w}_{1}}^{2}\left(\mathbf{w}_{1}, \mathbf{w}_{2}, \varsigma\right)+\mathbb{Q}_{\mathbf{w}_{2} \mathbf{w}_{2}}^{2}\left(\mathbf{w}_{1}, \mathbf{w}_{2}, \varsigma\right)+\hbar \mathbb{Q}\left(\mathbf{w}_{1}, \mathbf{w}_{2}, \varsigma\right)\left(1-r_{1} \mathbb{Q}\left(\mathbf{w}_{1}, \mathbf{w}_{2}, \varsigma\right)\right)\right]
$$

Employing the inverse generalized integral transform, we obtain

$\mathscr{Q}\left(\mathbf{w}_{1}, \mathbf{w}_{2}, \varsigma\right)=\mathbb{J}^{-1}\left[\frac{\psi(\mathfrak{g})}{\phi(\mathfrak{g})} \mathscr{Q}\left(\mathbf{w}_{1}, \mathbf{w}_{2}, 0\right)+\frac{1}{\psi^{\chi}(\mathfrak{g})} \mathbb{J}\left[\mathscr{Q}_{\mathbf{w}_{1} \mathbf{w}_{1}}^{2}\left(\mathbf{w}_{1}, \mathbf{w}_{2}, \varsigma\right)+\widehat{Q}_{\mathbf{w}_{2} \mathbf{w}_{2}}^{2}\left(\mathbf{w}_{1}, \mathbf{w}_{2}, \varsigma\right)+\hbar Q\left(\mathbf{w}_{1}, \mathbf{w}_{2}, \varsigma\right)\left(1-r_{1} Q\left(\mathbf{w}_{1}, \mathbf{w}_{2}, \varsigma\right)\right)\right]\right.$

Thanks to the generalized decomposition method, we find

$$
\begin{aligned}
\widehat{Q}_{0}\left(\mathbf{w}_{1}, \mathbf{w}_{2}, \varsigma\right) & =\mathbb{J}^{-1}\left[\frac{\psi(\mathfrak{g})}{\phi(\mathfrak{g})} \mathscr{Q}\left(\mathbf{w}_{1}, \mathbf{w}_{2}, 0\right)\right]=\mathbb{J}^{-1}\left[\frac{\psi(\mathfrak{g})}{\phi(\mathfrak{I})} \exp \left(\sqrt{\frac{\hbar r_{1}}{8}}\left(\mathbf{w}_{1}+\mathbf{w}_{2}\right)\right)\right] \\
& =\exp \left(\sqrt{\frac{\hbar r_{1}}{8}}\left(\mathbf{w}_{1}+\mathbf{w}_{2}\right)\right) .
\end{aligned}
$$

Here, we surmise that the unknown function $Q\left(\mathbf{w}_{1}, \mathbf{w}_{2}, \varsigma\right)$ can be written by an infinite series of the form

$$
Q\left(\mathbf{w}_{1}, \mathbf{w}_{2}, \varsigma\right)=\sum_{\ell=0}^{\infty} Q_{\ell}\left(\mathbf{w}_{1}, \mathbf{w}_{2}, \varsigma\right)
$$




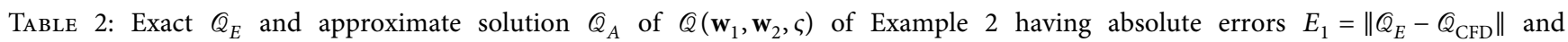
$E_{2}=\left\|Q_{E}-Q_{\mathrm{ABC}}\right\|$ when $\chi=1, \varsigma=0.2, r_{1}=-8 / 9$, and $\hbar=-1$ for different values of $\mathbf{w}_{1}$ and $\mathbf{w}_{2}$.

\begin{tabular}{|c|c|c|c|c|c|c|}
\hline$\left(\mathbf{w}_{1}, \mathbf{w}_{2}\right)$ & $\mathbb{Q}_{E}$ sol. & $\mathbb{Q}_{\text {CFD }}$ sol. & $\mathbb{Q}_{\mathrm{ABC}}$ sol. & VIMHP sol. [23] & Error $=E_{1}$ & Error $=E_{2}$ \\
\hline$(-10,10)$ & 10.303436 & 10.303000 & 10.302851 & 10.993451 & $5.8565 e-4$ & $5.8565 e-4$ \\
\hline$(-8,8)$ & 5.552607 & 5.552292 & 5.552292 & 5.990091 & $3.1561 e-4$ & $3.1561 e-4$ \\
\hline$(-6,6)$ & 1.455853 & 1.455770 & 1.455770 & 1.995099 & $8.2751 e-5$ & $8.2751 e-5$ \\
\hline$(-4,4)$ & 2.259085 & 2.258957 & 2.258957 & 2.879342 & $1.2841 e-4$ & $1.2841 e-4$ \\
\hline$(-2,2)$ & 1.391787 & 1.391708 & 1.391708 & 2.600834 & $7.9109 e-5$ & $7.9109 e-5$ \\
\hline$(0,0)$ & 0.000000 & 0.000000 & 0.000000 & 0.990087 & 0.000000 & 0.000000 \\
\hline$(2,2)$ & 1.391787 & 1.391700 & 1.391700 & 1.990980 & $7.9109 e-5$ & $7.9109 e-5$ \\
\hline$(4,4)$ & 2.259085 & 2.258957 & 2.258957 & 2.998456 & $1.2841 e-4$ & $1.2841 e-4$ \\
\hline$(6,6)$ & 1.455853 & 1.455770 & 1.455770 & 2.009987 & $8.2751 e-5$ & $8.2751 e-5$ \\
\hline$(8,8)$ & 5.552607 & 5.5523292 & 5.5523292 & 5.968901 & $3.1561 e-4$ & $3.1561 e-4$ \\
\hline$(10,10)$ & 10.303000 & 10.303044 & 10.303044 & 10.990234 & $5.8565 e-4$ & $5.8565 e-4$ \\
\hline
\end{tabular}

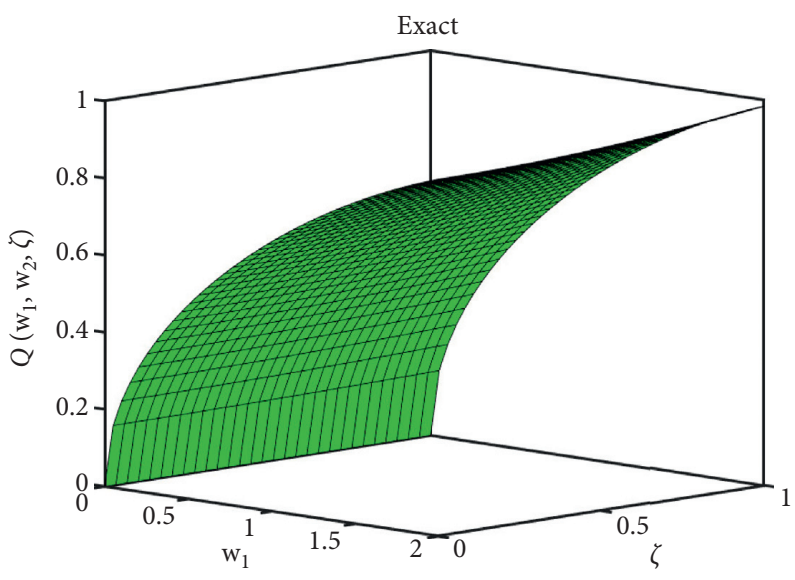

(a)

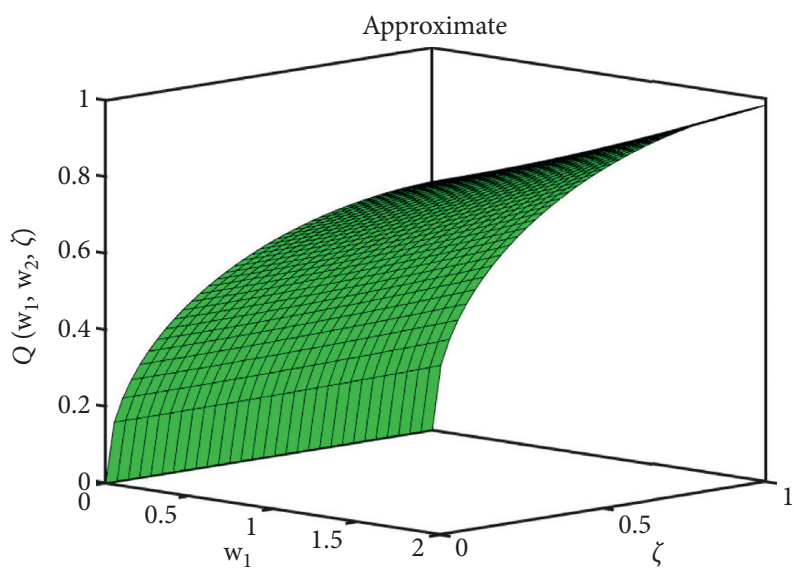

(b)

FIGURE 4: Three-dimensional illustration of exact and approximate solution of Example 2 when $\alpha=1, r_{1}=0, \varsigma=0.01, \hbar=1$, and $\chi=1$.

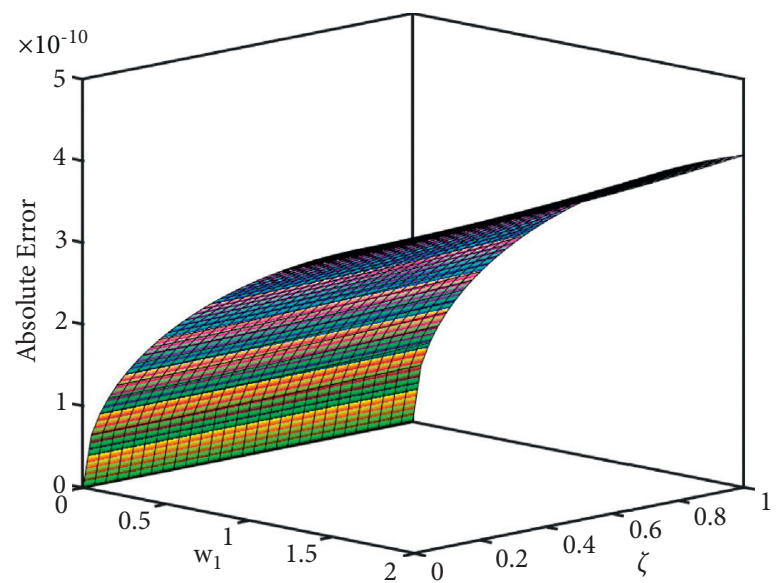

(a)

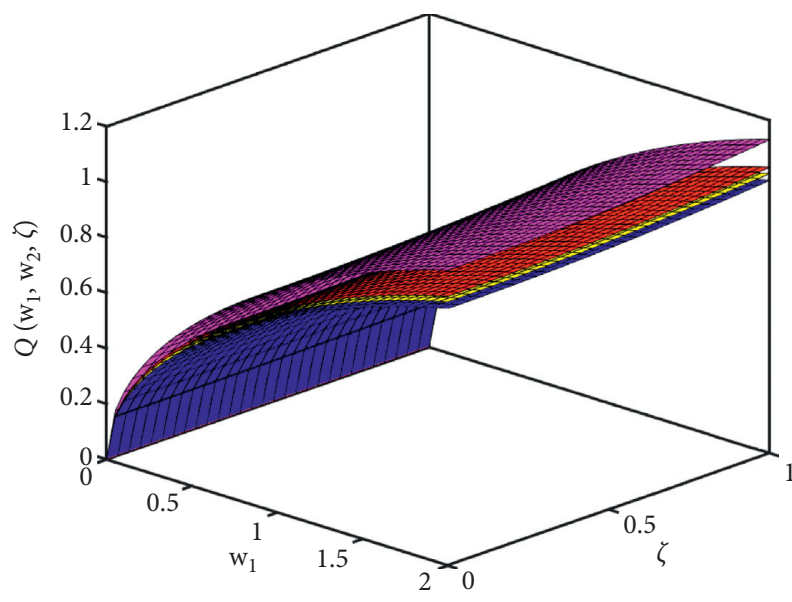

Exact

$x=1$

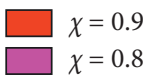

(b)

FIGURE 5: Three-dimensional illustration of the absolute error and multiple surface of various fractional orders for Example 2 when $\alpha=1, r_{1}=0, \hbar=1$, and $\varsigma=0.01$. 


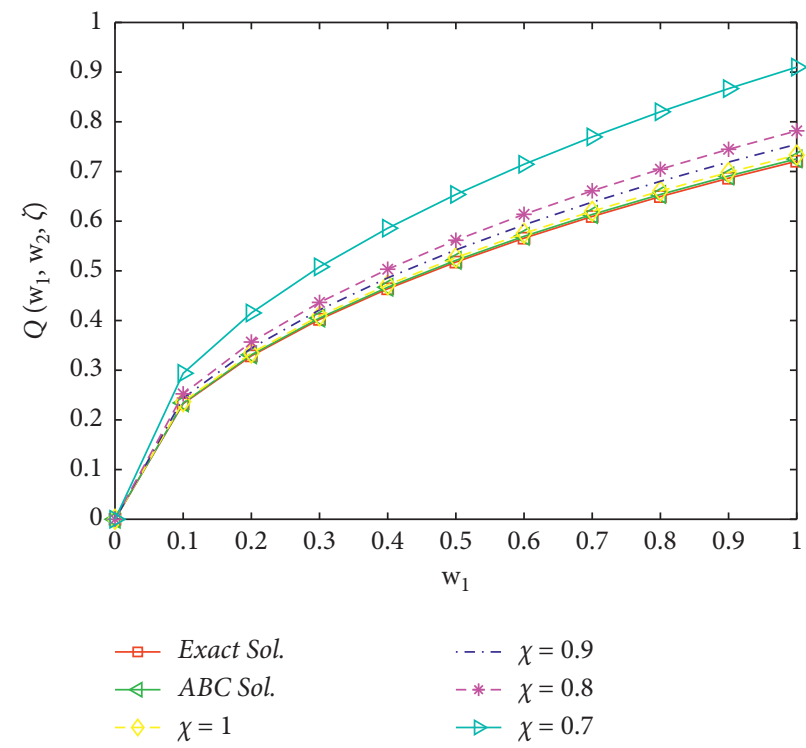

FIgURE 6: Two-dimensional illustration of Example 2 for various fractional orders when $\alpha=1, r_{1}=0, \hbar=1$, and $\varsigma=0.01$.

Also, the nonlinearity $\mathscr{F}_{j}(\mathscr{Q}), j=1,2,3$, can be $\infty^{\infty} \mathscr{B}_{\ell}$ defined in (44) along with decomposed by an infinite series of polynomials represented $\quad \mathscr{F}_{3}(\mathscr{Q})=\mathscr{Q}\left(1-r_{1} Q\right)=\sum_{\ell=0}^{\infty} \mathscr{C}_{\ell}$. Thus, we have by $\quad \mathscr{F}_{1}(\mathcal{Q})=Q_{\mathbf{w}_{1} \mathbf{w}_{1}}^{2}=\sum_{\ell=0}^{\infty} \mathscr{A}_{\ell}$ and $\mathscr{F}_{2}(Q)=Q_{\mathbf{w}_{2} \mathbf{w}_{2}}^{2}=\sum_{\ell=0}$

$$
\begin{aligned}
\mathscr{C}_{\ell}\left(\mathscr{Q}-r_{1} \mathscr{Q}^{2}\right)= \begin{cases}\left(\mathscr{Q}_{0}-r_{1} Q_{0}^{2}\right), & \ell=0, \\
\left(Q_{2}-r_{1} Q_{0} Q_{1}\right), & \ell=1, \\
\left(Q_{2}-2 r_{1} Q_{0} Q_{2}-r_{1} Q_{1}^{2}\right), & \ell=2,\end{cases} \\
\sum_{\ell=0}^{\infty} \mathscr{Q}_{\ell+1}\left(\mathbf{w}_{1}, \mathbf{w}_{2}, \varsigma\right)=\mathbb{J}^{-1}\left[\frac{1}{\psi^{\chi}(\mathfrak{T})} \sqrt{ }\left[\sum_{\ell=0}^{\infty}(\mathscr{A})_{\ell}+\sum_{\ell=0}^{\infty}(\mathscr{B})_{\ell}+\sum_{\ell=0}^{\infty}(\mathscr{C})_{\ell}\right], \quad \ell=0,1,2, \ldots\right.
\end{aligned}
$$

For $\ell=0,1,2,3, \ldots$,

$$
\begin{aligned}
\mathscr{Q}_{1}\left(\mathbf{w}_{1}, \mathbf{w}_{2}, \varsigma\right) & =\mathbb{J}^{-1}\left[\frac{1}{\psi^{\chi}(\mathfrak{g})} \mathbb{J}\left[\mathscr{A}_{0}+\mathscr{B}_{0}+\mathscr{C}_{0}\right]\right] \\
& =\exp \left(\sqrt{\frac{\hbar r_{1}}{8}}\left(\mathbf{w}_{1}+\mathbf{w}_{2}\right)\right) \frac{\varsigma^{\chi}}{\Gamma(\chi+1)}, \\
\mathbb{Q}_{2}\left(\mathbf{w}_{1}, \mathbf{w}_{2}, \varsigma\right) & =\mathbb{J}^{-1}\left[\frac{1}{\psi^{\chi}(\mathfrak{g})} \mathbb{J}\left[\mathscr{A}_{1}+\mathscr{B}_{1}+\mathscr{C}_{1}\right]\right] \\
& =\exp \left(\sqrt{\frac{\hbar r_{1}}{8}}\left(\mathbf{w}_{1}+\mathbf{w}_{2}\right)\right) \frac{\varsigma^{2 \chi}}{\Gamma(2 \chi+1)}, \\
\mathbb{Q}_{3}\left(\mathbf{w}_{1}, \mathbf{w}_{2}, \varsigma\right) & =\mathbb{J}^{-1}\left[\frac{1}{\psi^{\chi}(\mathfrak{g})} \mathbb{J}\left[\mathscr{A}_{2}+\mathscr{B}_{2}+\mathscr{C}_{2}\right]\right] \\
& =\exp \left(\sqrt{\frac{\hbar r_{1}}{8}}\left(\mathbf{w}_{1}+\mathbf{w}_{2}\right)\right) \frac{\varsigma^{3 \chi}}{\Gamma(3 \chi+1)},
\end{aligned}
$$


The approximate solution for Example 3 is expressed as follows:

$$
\begin{aligned}
Q\left(\mathbf{w}_{1}, \mathbf{w}_{2}, \varsigma\right) & =\Phi_{0}\left(\mathbf{w}_{1}, \mathbf{w}_{2}, \varsigma\right)+\Phi_{1}\left(\mathbf{w}_{1}, \mathbf{w}_{2}, \varsigma\right)+\Phi_{2}\left(\mathbf{w}_{1}, \mathbf{w}_{2}, \varsigma\right)+\Phi_{3}\left(\mathbf{w}_{1}, \mathbf{w}_{2}, \varsigma\right)+\cdots \\
& =\exp \left(\sqrt{\frac{\hbar r_{1}}{8}}\left(\mathbf{w}_{1}+\mathbf{w}_{2}\right)\right)\left(1+\frac{\varsigma^{\chi}}{\Gamma(\chi+1)}+\frac{\varsigma^{2 \chi}}{\Gamma(2 \chi+1)}+\frac{\varsigma^{3 \chi}}{\Gamma(3 \chi+1)}+\cdots\right)
\end{aligned}
$$

Case 2. Here, we surmise ABC fractional derivative operator coupled with the generalized integral transform and
Adomian decomposition method. Applying the generalized integral transform for Example 3,

$$
\begin{aligned}
\frac{\psi^{\chi}(\mathfrak{S}) \mathbb{A}(\chi)}{\chi+(1-\chi) \psi^{\chi}(\mathfrak{g})} \mathscr{U}\left(\mathbf{w}_{1}, \mathfrak{g}\right)-\phi(\mathfrak{g}) \sum_{\kappa=0}^{m-1} \psi^{\chi-\kappa-1}(\mathfrak{g}) \mathbb{Q}^{(\kappa)}(0)= & \mathbb{J}\left[\mathcal{Q}_{\mathbf{w}_{1} \mathbf{w}_{1}}^{2}\left(\mathbf{w}_{1}, \mathbf{w}_{2}, \varsigma\right)+\mathcal{Q}_{\mathbf{w}_{2} \mathbf{w}_{2}}^{2}\left(\mathbf{w}_{1}, \mathbf{w}_{2}, \varsigma\right)\right. \\
& \left.+\hbar \mathbb{Q}\left(\mathbf{w}_{1}, \mathbf{w}_{2}, \varsigma\right)\left(1-r_{1} \mathbb{Q}\left(\mathbf{w}_{1}, \mathbf{w}_{2}, \varsigma\right)\right)\right] .
\end{aligned}
$$

Taking into consideration the IC given in (77), we have

$$
\begin{aligned}
\mathscr{U}\left(\mathbf{w}_{1}, \mathfrak{g}\right)= & \frac{\psi(\mathfrak{I})}{\phi(\mathfrak{g})} \mathscr{Q}\left(\mathbf{w}_{1}, \mathbf{w}_{2}, 0\right)+\frac{\chi+(1-\chi) \psi^{\chi}(\mathfrak{g})}{\psi^{\chi}(\mathfrak{I}) \mathbb{A}(\chi)} \mathbb{J}\left[\widehat{Q}_{\mathbf{w}_{1} \mathbf{w}_{1}}^{2}\left(\mathbf{w}_{1}, \mathbf{w}_{2}, \varsigma\right)+\mathscr{Q}_{\mathbf{w}_{2} \mathbf{w}_{2}}^{2}\left(\mathbf{w}_{1}, \mathbf{w}_{2}, \varsigma\right)\right. \\
& \left.+\hbar \mathscr{Q}\left(\mathbf{w}_{1}, \mathbf{w}_{2}, \varsigma\right)\left(1-r_{1} \mathscr{Q}\left(\mathbf{w}_{1}, \mathbf{w}_{2}, \varsigma\right)\right)\right] .
\end{aligned}
$$

Employing the inverse generalized integral transform, we obtain

$$
\begin{aligned}
\mathscr{Q}\left(\mathbf{w}_{1}, \mathbf{w}_{2}, \varsigma\right)= & \mathbb{J}^{-1}\left[\frac{\psi(\mathfrak{I})}{\phi(\mathfrak{I})} \mathscr{Q}\left(\mathbf{w}_{1}, \mathbf{w}_{2}, 0\right)+\frac{\chi+(1-\chi) \psi^{\chi}(\mathfrak{I})}{\psi^{\chi}(\mathfrak{I}) \mathbb{A}(\chi)} \mathbb{J}\left[\mathcal{Q}_{\mathbf{w}_{1} \mathbf{w}_{1}}^{2}\left(\mathbf{w}_{1}, \mathbf{w}_{2}, \varsigma\right)+\mathscr{Q}_{\mathbf{w}_{2} \mathbf{w}_{2}}^{2}\left(\mathbf{w}_{1}, \mathbf{w}_{2}, \varsigma\right)\right.\right. \\
& \left.+\hbar \mathscr{Q}\left(\mathbf{w}_{1}, \mathbf{w}_{2}, \varsigma\right)\left(1-r_{1} \mathscr{Q}\left(\mathbf{w}_{1}, \mathbf{w}_{2}, \varsigma\right)\right)\right] .
\end{aligned}
$$
find

Thanks to the generalized decomposition method, we

$$
\begin{aligned}
& \begin{aligned}
\mathbb{Q}_{0}\left(\mathbf{w}_{1}, \mathbf{w}_{2}, \varsigma\right) & =\mathbb{J}^{-1}\left[\frac{\psi(\mathfrak{g})}{\phi(\mathfrak{g})} \mathscr{Q}\left(\mathbf{w}_{1}, \mathbf{w}_{2}, 0\right)\right]=\mathbb{J}^{-1}\left[\frac{\psi(\mathfrak{g})}{\phi(\mathfrak{g})} \exp \left(\sqrt{\frac{\hbar r_{1}}{8}}\left(\mathbf{w}_{1}+\mathbf{w}_{2}\right)\right)\right] \\
& =\exp \left(\sqrt{\frac{\hbar r_{1}}{8}}\left(\mathbf{w}_{1}+\mathbf{w}_{2}\right)\right) . \\
& \mathbb{Q}\left(\mathbf{w}_{1}, \mathbf{w}_{2}, \varsigma\right)=\sum_{\ell=0}^{\infty} \mathbb{Q}_{\ell}\left(\mathbf{w}_{1}, \mathbf{w}_{2}, \varsigma\right) .
\end{aligned} \\
& \text { written by an infinite series of the form }
\end{aligned}
$$

Here, we surmise that the unknown function $Q\left(\mathbf{w}_{1}, \mathbf{w}_{2}, \varsigma\right)$ can be written by an infinite series of the form 
Also, the nonlinearity $\mathscr{F}_{j}(\mathscr{Q}), j=1,2,3$, can be decomposed by an infinite series of polynomials represented by $\quad \mathscr{F}_{1}(\mathscr{Q})=Q_{\mathbf{w}_{1} \mathbf{w}_{1}}^{2}=\sum_{\ell=0}^{\infty} \mathscr{A}_{\ell}$ and $\mathscr{F}_{2}(\mathscr{Q})=Q_{\mathbf{w}_{2} \mathbf{w}_{2}}^{2}$ $=\sum_{\ell=0}^{\infty} \mathscr{B}_{\ell}$ along with $\mathscr{F}_{3}(\mathscr{Q})=\mathscr{Q}\left(1-r_{1} \mathscr{Q}\right)=\sum_{\ell=0}^{\infty} \mathscr{C}_{\ell}$ defined in (44) and (83), respectively.

For $\ell=0,1,2,3, \ldots$,

$$
\begin{aligned}
\mathscr{Q}_{1}\left(\mathbf{w}_{1}, \mathbf{w}_{2}, \varsigma\right) & =\mathbb{J}^{-1}\left[\frac{\chi+(1-\chi) \psi^{\chi}(\mathfrak{g})}{\psi^{\chi}(\mathfrak{g}) \mathbb{A}(\chi)} \mathbb{I}\left[\mathscr{A}_{0}+\mathscr{B}_{0}+\mathscr{C}_{0}\right]\right] \\
& =\frac{1}{\mathbb{A}(\chi)} \exp \left(\sqrt{\frac{\hbar r_{1}}{8}}\left(\mathbf{w}_{1}+\mathbf{w}_{2}\right)\right)\left[\frac{\chi \varsigma^{\chi}}{\Gamma(\chi+1)}+(1-\chi)\right], \\
\mathbb{Q}_{2}\left(\mathbf{w}_{1}, \mathbf{w}_{2}, \varsigma\right) & =\mathbb{J}^{-1}\left[\frac{\chi+(1-\chi) \psi^{\chi}(\mathfrak{g})}{\psi^{\chi}(\mathfrak{B}) \mathbb{A}(\chi)} \mathbb{I}\left[\mathscr{A}_{1}+\mathscr{B}_{1}+\mathscr{C}_{1}\right]\right] \\
& =\frac{1}{\mathbb{A}^{2}(\chi)} \exp \left(\sqrt{\frac{\hbar r_{1}}{8}}\left(\mathbf{w}_{1}+\mathbf{w}_{2}\right)\right)\left[\frac{\chi^{2} \varsigma^{2 \chi}}{\Gamma(2 \chi+1)}+2 \chi(1-\chi) \frac{\varsigma^{\chi}}{\Gamma(\chi+1)}+(1-\chi)^{2}\right],
\end{aligned}
$$

$$
\begin{aligned}
\mathscr{Q}_{3}\left(\mathbf{w}_{1}, \mathbf{w}_{2}, \varsigma\right) & =\mathbb{J}^{-1}\left[\frac{\chi+(1-\chi) \psi^{\chi}(\mathfrak{g})}{\psi^{\chi}(\mathfrak{B}) \mathbb{A}(\chi)} \mathbb{D}\left[\mathscr{A}_{2}+\mathscr{B}_{2}+\mathscr{C}_{2}\right]\right] \\
& =\frac{1}{\mathbb{A}^{3}(\chi)} \exp \left(\sqrt{\frac{\hbar r_{1}}{8}}\left(\mathbf{w}_{1}+\mathbf{w}_{2}\right)\right)\left[\frac{\chi^{3} \varsigma^{3 \chi}}{\Gamma(3 \chi+1)}+3 \chi^{2}(1-\chi) \frac{\varsigma^{2 \chi}}{\Gamma(2 \chi+1)}+3 \chi(1-\chi)^{2} \frac{\varsigma^{\chi}}{\Gamma(\chi+1)}+(1-\chi)^{3}\right],
\end{aligned}
$$$$
\vdots
$$

The approximate solution for Example 3 is expressed as follows:

$$
\begin{aligned}
\mathbb{Q}\left(\mathbf{w}_{1}, \mathbf{w}_{2}, \varsigma\right)= & \Phi_{0}\left(\mathbf{w}_{1}, \mathbf{w}_{2}, \varsigma\right)+\Phi_{1}\left(\mathbf{w}_{1}, \mathbf{w}_{2}, \varsigma\right)+\Phi_{2}\left(\mathbf{w}_{1}, \mathbf{w}_{2}, \varsigma\right)+\Phi_{3}\left(\mathbf{w}_{1}, \mathbf{w}_{2}, \varsigma\right)+\cdots \\
= & \exp \left(\sqrt{\frac{\hbar r_{1}}{8}}\left(\mathbf{w}_{1}+\mathbf{w}_{2}\right)\right)\left(1+\frac{1}{\mathbb{A}(\chi)}\left(\frac{\varsigma^{\chi}}{\Gamma(\chi+1)}+(1-\chi)\right)\right. \\
& +\frac{1}{\mathbb{A}^{2}(\chi)}\left(\frac{\chi^{2} \varsigma^{2 \chi}}{\Gamma(2 \chi+1)}+2 \chi(1-\chi) \frac{\varsigma^{\chi}}{\Gamma(\chi+1)}+(1-\chi)^{2}\right) \\
& \left.+\frac{1}{\mathbb{A}^{3}(\chi)}\left(\frac{\chi^{3} \varsigma^{3 \chi}}{\Gamma(3 \chi+1)}+3 \chi^{2}(1-\chi) \frac{\varsigma^{2 \chi}}{\Gamma(2 \chi+1)}+3 \chi(1-\chi)^{2} \frac{\varsigma^{\chi}}{\Gamma(\chi+1)}+(1-\chi)^{3}\right)+\cdots\right) .
\end{aligned}
$$

For $\chi=1$, we obtained the exact solution of Example 3 as

$$
\mathcal{Q}\left(\mathbf{w}_{1}, \mathbf{w}_{2}, \varsigma\right)=\exp \left(\sqrt{\frac{\hbar r_{1}}{8}}\left(\mathbf{w}_{1}+\mathbf{w}_{2}\right)+\varsigma\right) .
$$

Table 3 shows the analytical approximate solutions with some free parameters that are provided by the proposed technique. The comparison analysis is conducted with the VIMHP that predicts the preciseness of the suggested scheme due to their lower error. The analytical findings are extremely useful in deciphering the internal components of acts of nature. The exact and numerical solution for $\alpha=1=\beta, \varsigma=0.01$, and $\chi=1$ is shown in Figure 7 .

Furthermore, the absolute error for the aforementioned assumptions is depicted in Figure 8(a). Figure 8(b) demonstrates the behavior of the findings in three-dimensional simulation for different fractional orders by employing the Caputo fractional derivative operator.

Finally, Figure 9 represents the two-dimensional behavior of exact, approximate by Caputo, and approximate solutions derived by $\mathrm{ABC}$ fractional operators with varying fractional orders. These solutions have a distinctive 
characteristic that allows them to interact with other solutions derived by [23]. The proposed findings have particlelike geometries in their solutions. The synthesized trajectory is either a success or a descent from one asymptotic state to the next. The accuracy of the proposed method can be enhanced by increasing the recursive terms.

Example 4. Assume the time-fractional BPM (4) having $\alpha=-1, \beta=1, \hbar=(1 / 96)$, and $r_{1}=48$; then,

$$
\mathbf{D}_{\zeta}^{\alpha} \mathscr{Q}\left(\mathbf{w}_{1}, \mathbf{w}_{2}, \varsigma\right)=Q_{\mathbf{w}_{1} \mathbf{w}_{1}}^{2}\left(\mathbf{w}_{1}, \mathbf{w}_{2}, \varsigma\right)+Q_{\mathbf{w}_{2} \mathbf{w}_{2}}^{2}\left(\mathbf{w}_{1}, \mathbf{w}_{2}, \varsigma\right)+\frac{1}{96} Q^{-1}\left(\mathbf{w}_{1}, \mathbf{w}_{2}, \varsigma\right)-\frac{1}{2},
$$

with IC

$$
Q_{0}\left(\mathbf{w}_{1}, \mathbf{w}_{2}, 0\right)=\frac{1}{4} \sqrt{2\left(\mathbf{w}_{1}^{2}+\mathbf{w}_{2}^{2}\right)+\mathbf{w}_{2}+5}
$$

Case 1. First, we apply the Caputo fractional derivative operator coupled with the generalized integral transform and Adomian decomposition method. Applying the generalized integral transform for Example 4,

Proof. Foremost, we provide the solution of (76) in two general cases.

$$
\psi^{\chi}(\mathfrak{g}) \mathcal{U}\left(\mathbf{w}_{1}, \mathfrak{G}\right)-\phi(\mathfrak{g}) \sum_{\kappa=0}^{m-1} \psi^{\chi-\kappa-1}(\mathfrak{g}) Q^{(\kappa)}(0)=\mathbb{J}\left[\mathbb{Q}_{\mathbf{w}_{1} \mathbf{w}_{1}}^{2}\left(\mathbf{w}_{1}, \mathbf{w}_{2}, \varsigma\right)+\mathbb{Q}_{\mathbf{w}_{2} \mathbf{w}_{2}}^{2}\left(\mathbf{w}_{1}, \mathbf{w}_{2}, \varsigma\right)+\frac{1}{96 Q_{0}}-\frac{1}{2}\right]
$$

Taking into consideration the IC given in (95), we have

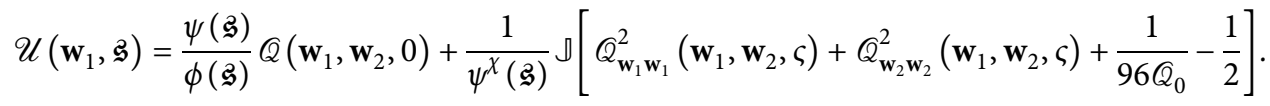

Employing the inverse generalized integral transform, we obtain

$$
\mathcal{Q}\left(\mathbf{w}_{1}, \mathbf{w}_{2}, \varsigma\right)=\mathbb{J}^{-1}\left[\frac{\psi(\mathfrak{g})}{\phi(\mathfrak{g})} \mathcal{Q}\left(\mathbf{w}_{1}, \mathbf{w}_{2}, 0\right)+\frac{1}{\psi^{\chi}(\mathfrak{g})} \sqrt{ }\left[\mathcal{Q}_{\mathbf{w}_{1} \mathbf{w}_{1}}^{2}\left(\mathbf{w}_{1}, \mathbf{w}_{2}, \varsigma\right)+Q_{\mathbf{w}_{2} \mathbf{w}_{2}}^{2}\left(\mathbf{w}_{1}, \mathbf{w}_{2}, \varsigma\right)+\frac{1}{96 Q_{0}}-\frac{1}{2}\right]\right]
$$
find

Thanks to the generalized decomposition method, we

$$
\begin{aligned}
& \qquad \begin{aligned}
\mathscr{Q}_{0}\left(\mathbf{w}_{1}, \mathbf{w}_{2}, \varsigma\right) & =\mathbb{J}^{-1}\left[\frac{\psi(\mathfrak{I})}{\phi(\mathfrak{g})} \mathscr{Q}\left(\mathbf{w}_{1}, \mathbf{w}_{2}, 0\right)\right]=\mathbb{J}^{-1}\left[\frac{\psi(\mathfrak{I})}{\phi(\mathfrak{g})} \frac{1}{4} \sqrt{2\left(\mathbf{w}_{1}^{2}+\mathbf{w}_{2}^{2}\right)+\mathbf{w}_{2}+5}\right] \\
& =\frac{1}{4} \sqrt{2\left(\mathbf{w}_{1}^{2}+\mathbf{w}_{2}^{2}\right)+\mathbf{w}_{2}+5}
\end{aligned} \\
& \qquad \mathbb{Q}\left(\mathbf{w}_{1}, \mathbf{w}_{2}, \varsigma\right)=\sum_{\ell=0}^{\infty} Q_{\ell}\left(\mathbf{w}_{1}, \mathbf{w}_{2}, \varsigma\right) .
\end{aligned}
$$

Here, we surmise that the unknown function $Q\left(\mathbf{w}_{1}, \mathbf{w}_{2}, \varsigma\right)$ can be written by an infinite series of the form 
TABLE 3: Exact $\mathscr{Q}_{E}$ and approximate solution $\mathbb{Q}_{A}$ of $\mathbb{Q}\left(\mathbf{w}_{1}, \mathbf{w}_{2}, \varsigma\right)$ of Example 3 having absolute errors $E_{1}=\left\|\mathbb{Q}_{E}-\mathbb{Q}_{\mathrm{CFD}}\right\|$ and $E_{2}=\left\|Q_{E}-Q_{A B C}\right\|$ when $\chi=1, \varsigma=0.2, r_{1}=-8 / 9$, and $\hbar=-1$ for different values of $\mathbf{w}_{1}$ and $\mathbf{w}_{2}$.

\begin{tabular}{|c|c|c|c|c|c|c|}
\hline$\left(\mathbf{w}_{1}, \mathbf{w}_{2}\right)$ & $\mathbb{Q}_{E}$ sol. & $\mathbb{Q}_{\text {CFD }}$ sol. & $\mathbb{Q}_{\mathrm{ABC}}$ sol. & VIMHP sol. [23] & Error $=E_{1}$ & Error $=E_{2}$ \\
\hline$(-10,10)$ & $1.04194 e-3$ & $1.04185 e-3$ & $1.041824 e-3$ & $1.04186 e-3$ & $8.1559 e-8$ & $8.1599 e-8$ \\
\hline$(-8,8)$ & $3.95279 e-3$ & $3.95249 e-3$ & $3.95243 e-3$ & $3.95248 e-3$ & $3.0941 e-7$ & $3.0939 e-7$ \\
\hline$(-6,6)$ & $1.49956 e-2$ & $1.49940 e-2$ & $1.49939 e-2$ & $1.49944 e-2$ & $1.1732 e-6$ & $1.1730 e-6$ \\
\hline$(-4,4)$ & $5.68882 e-2$ & $5.68832 e-2$ & $5.68830 e-2$ & $5.68838 e-2$ & $4.4529 e-6$ & $4.4527 e-6$ \\
\hline$(-2,2)$ & $2.15815 e-1$ & $2.15792 e-1$ & $2.15790 e-1$ & $2.15798 e-1$ & $1.6893 e-5$ & $1.6890 e-5$ \\
\hline$(0,0)$ & $8.18731 e-1$ & $8.186677 e-1$ & $8.186670 e-1$ & $8.186667 e-1$ & $6.4086 e-5$ & $6.4082 e-5$ \\
\hline$(2,2)$ & 3.10599000 & 3.10575 & 3.10570 & 3.10566 & $2.4312 e-4$ & $2.4310 e-4$ \\
\hline$(4,4)$ & 11.7831000 & 11.78212 & 11.78210 & 11.78218 & $9.2233 e-4$ & $9.2226 e-4$ \\
\hline$(6,6)$ & 44.7011800 & 44.69756 & 44.69750 & 44.69769 & $3.4989 e-3$ & $3.4982 e-3$ \\
\hline$(8,8)$ & 169.581450 & 169.56803 & 169.56810 & 169.56817 & $1.3274 e-2$ & $1.3278 e-2$ \\
\hline$(10,10)$ & 643.335670 & 643.28526 & 643.28519 & 643.28534 & $5.0357 e-2$ & $5.0350 e-2$ \\
\hline
\end{tabular}

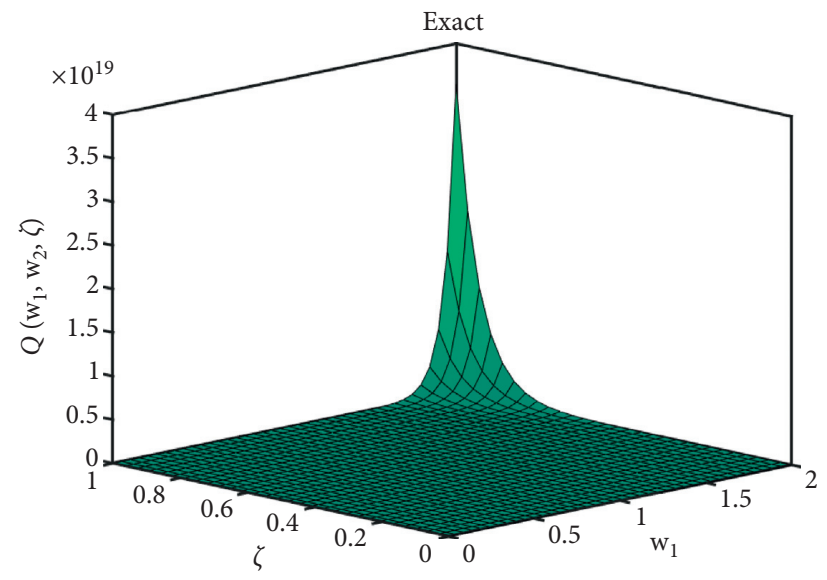

(a)

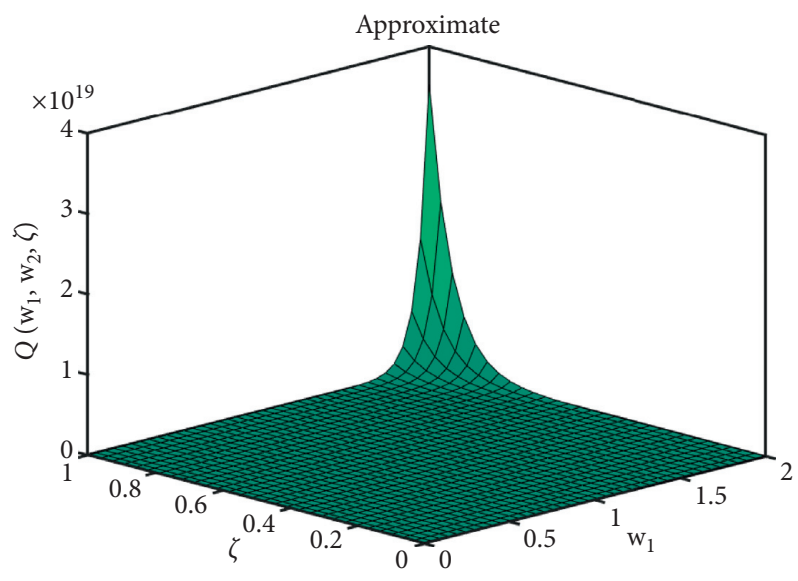

(b)

FIgURE 7: Three-dimensional illustration of exact and approximate solution of Example 3 when $\alpha=1=\beta, r_{1}=0, \varsigma=0.01, \hbar=1$, and $\chi=1$.

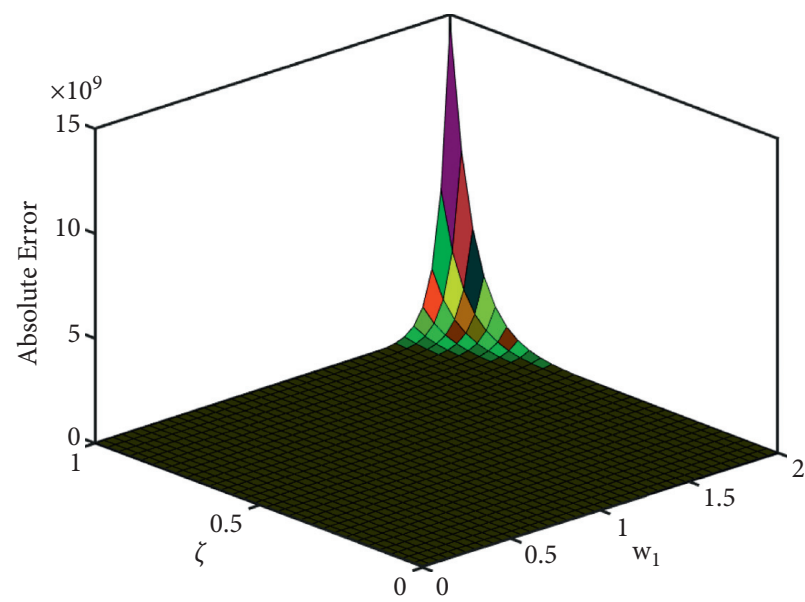

(a)

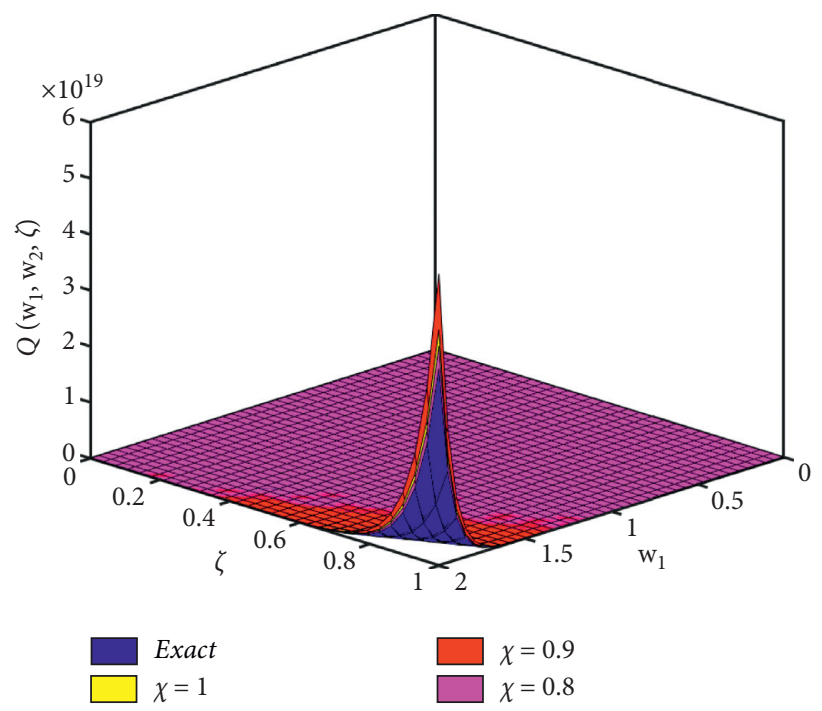

(b)

Figure 8: Three-dimensional illustration of the absolute error and multiple surface of various fractional orders for Example 3 when $\alpha=1=\beta, r_{1}=0, \hbar=1$, and $\varsigma=0.01$. 


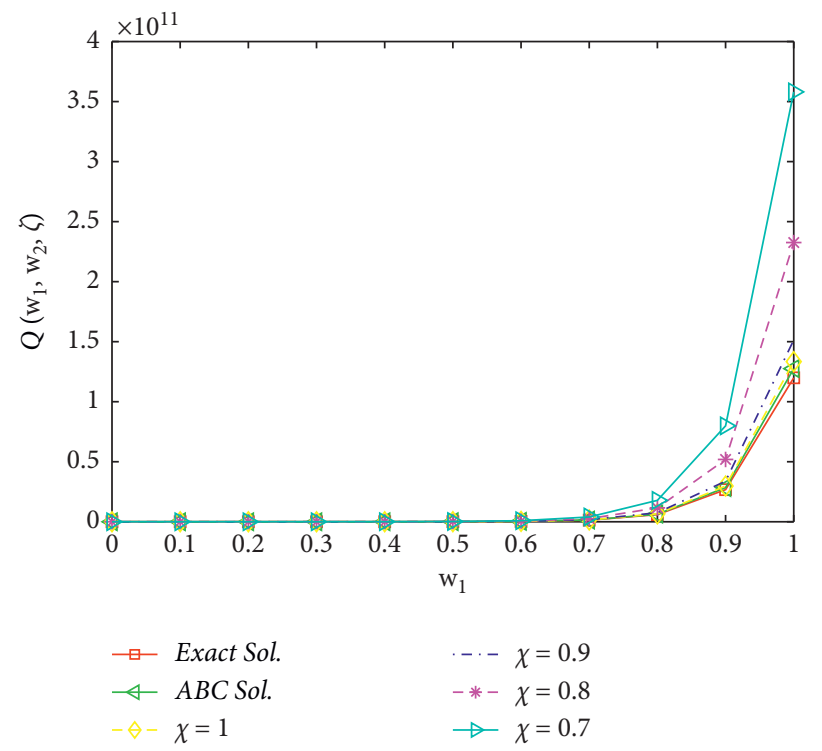

Figure 9: Two-dimensional illustration of Example 3 for various fractional orders when $\alpha=1=\beta, r_{1}=0, \hbar=1$, and $\varsigma=0.01$.

Also, the nonlinearity $\mathscr{F}_{j}(\mathscr{Q}), j=1,2,3$, can be $\sum_{\ell=0}^{\infty} \mathscr{B}_{\ell}$ defined in (44) along with decomposed by an infinite series of polynomials represented $\quad \mathscr{F}_{3}(\mathscr{Q})=\left(1 / 96 \mathbb{Q}_{0}\right)-(1 / 2)=\sum_{\ell=0}^{\infty} \mathscr{D}_{\ell}$. Thus, we have by $\quad \mathscr{F}_{1}(Q)=Q_{\mathbf{w}_{1} \mathbf{w}_{1}}^{2}=\sum_{\ell=0}^{\infty} \mathscr{A}_{\ell}$ and $\mathscr{F}_{2}(\mathbb{Q})=\mathbb{Q}_{\mathbf{w}_{2} \mathbf{w}_{2}}^{2}=$

$$
\begin{aligned}
& \mathscr{D}_{\ell}\left(\frac{1}{96 Q}-\frac{1}{2}\right)= \begin{cases}\left(\frac{1}{96 Q_{0}}-\frac{1}{2}\right), & \ell=0, \\
-\frac{1}{96}\left(\frac{\mathscr{Q}_{1}}{\mathscr{Q}_{0}^{2}}\right), & \ell=1, \\
+\frac{1}{96}\left(\frac{-Q_{2}}{\mathscr{Q}_{0}^{2}}+\frac{Q_{1}^{2}}{\mathscr{Q}_{0}^{3}}\right), & \ell=2,\end{cases} \\
& \sum_{\ell=0}^{\infty} Q_{\ell+1}\left(\mathbf{w}_{1}, \mathbf{w}_{2}, \varsigma\right)=\mathbb{J}^{-1}\left[\frac{1}{\psi^{X}(\mathfrak{G})} \mathbb{J}\left[\sum_{\ell=0}^{\infty}(\mathscr{A})_{\ell}+\sum_{\ell=0}^{\infty}(\mathscr{B})_{\ell}+\sum_{\ell=0}^{\infty}(\mathscr{D})_{\ell}\right]\right], \quad \ell=0,1,2, \ldots
\end{aligned}
$$

For $\ell=0,1,2,3, \ldots$, 


$$
\begin{aligned}
\mathbb{Q}_{1}\left(\mathbf{w}_{1}, \mathbf{w}_{2}, \varsigma\right) & =\mathbb{J}^{-1}\left[\frac{1}{\psi^{\chi}(\mathfrak{g})} \mathbb{J}\left[\mathscr{A}_{0}+\mathscr{B}_{0}+\mathscr{D}_{0}\right]\right] \\
& =\frac{1}{24}\left(2\left(\mathbf{w}_{1}^{2}+\mathbf{w}_{2}^{2}+\mathbf{w}_{2}+5\right)\right)^{-1 / 2} \frac{\varsigma^{\chi}}{\Gamma(\chi+1)}, \\
\mathbb{Q}_{2}\left(\mathbf{w}_{1}, \mathbf{w}_{2}, \varsigma\right) & =\mathbb{J}^{-1}\left[\frac{1}{\psi^{\chi}(\mathfrak{g})} \mathbb{I}\left[\mathscr{A}_{1}+\mathscr{B}_{1}+\mathscr{D}_{1}\right]\right] \\
& =-\frac{1}{144}\left(2\left(\mathbf{w}_{1}^{2}+\mathbf{w}_{2}^{2}+\mathbf{w}_{2}+5\right)\right)^{-3 / 2} \frac{\varsigma^{2 \chi}}{\Gamma(2 \chi+1)}, \\
\mathbb{Q}_{3}\left(\mathbf{w}_{1}, \mathbf{w}_{2}, \varsigma\right) & =\mathbb{J}^{-1}\left[\frac{1}{\psi^{\chi}(\mathfrak{g})} \mathbb{I}\left[\mathscr{A}_{2}+\mathscr{B}_{2}+\mathscr{D}_{2}\right]\right] \\
& =\frac{1}{288}\left(2\left(\mathbf{w}_{1}^{2}+\mathbf{w}_{2}^{2}+\mathbf{w}_{2}+5\right)\right)^{-5 / 2} \frac{\varsigma^{3 \chi}}{\Gamma(3 \chi+1)},
\end{aligned}
$$

The approximate solution for Example 3 is expressed as follows:

$$
\begin{aligned}
Q\left(\mathbf{w}_{1}, \mathbf{w}_{2}, \varsigma\right)= & \Phi_{0}\left(\mathbf{w}_{1}, \mathbf{w}_{2}, \varsigma\right)+\Phi_{1}\left(\mathbf{w}_{1}, \mathbf{w}_{2}, \varsigma\right)+\Phi_{2}\left(\mathbf{w}_{1}, \mathbf{w}_{2}, \varsigma\right)+\Phi_{3}\left(\mathbf{w}_{1}, \mathbf{w}_{2}, \varsigma\right)+\cdots \\
= & \frac{1}{4} \sqrt{2\left(\mathbf{w}_{1}^{2}+\mathbf{w}_{2}^{2}\right)+\mathbf{w}_{2}+5}+\frac{1}{24}\left(2\left(\mathbf{w}_{1}^{2}+\mathbf{w}_{2}^{2}+\mathbf{w}_{2}+5\right)\right)^{-1 / 2} \frac{\varsigma^{\chi}}{\Gamma(\chi+1)} \\
& -\frac{1}{144}\left(2\left(\mathbf{w}_{1}^{2}+\mathbf{w}_{2}^{2}+\mathbf{w}_{2}+5\right)\right)^{-3 / 2} \frac{\varsigma^{2 \chi}}{\Gamma(2 \chi+1)}+\frac{1}{288}\left(2\left(\mathbf{w}_{1}^{2}+\mathbf{w}_{2}^{2}+\mathbf{w}_{2}+5\right)\right)^{-5 / 2} \frac{\varsigma^{3 \chi}}{\Gamma(3 \chi+1)}+\cdots
\end{aligned}
$$

Case 2. Here, we surmise ABC fractional derivative operator coupled with the generalized integral transform and
Adomian decomposition method. Applying the generalized integral transform for Example 4,

$$
\frac{\psi^{\chi}(\mathfrak{g}) \mathbb{A}(\chi)}{\chi+(1-\chi) \psi^{\chi}(\mathfrak{g})} \mathscr{U}\left(\mathbf{w}_{1}, \mathfrak{g}\right)-\phi(\mathfrak{g}) \sum_{\kappa=0}^{m-1} \psi^{\chi-\kappa-1}(\mathfrak{g}) \mathbb{Q}^{(\kappa)}(0)=\mathbb{J}\left[\mathbb{Q}_{\mathbf{w}_{1} \mathbf{w}_{1}}^{2}\left(\mathbf{w}_{1}, \mathbf{w}_{2}, \varsigma\right)+Q_{\mathbf{w}_{2} \mathbf{w}_{2}}^{2}\left(\mathbf{w}_{1}, \mathbf{w}_{2}, \varsigma\right)+\frac{1}{96 Q_{0}}-\frac{1}{2}\right]
$$

Taking into consideration the IC given in (95), we have

$$
\mathcal{U}\left(\mathbf{w}_{1}, \mathfrak{g}\right)=\frac{\psi(\mathfrak{g})}{\phi(\mathfrak{g})} \mathscr{Q}\left(\mathbf{w}_{1}, \mathbf{w}_{2}, 0\right)+\frac{\chi+(1-\chi) \psi^{\chi}(\mathfrak{g})}{\psi^{\chi}(\mathfrak{g}) \mathbb{A}(\chi)} \mathbb{J}\left[\mathcal{Q}_{\mathbf{w}_{1} \mathbf{w}_{1}}^{2}\left(\mathbf{w}_{1}, \mathbf{w}_{2}, \varsigma\right)+Q_{\mathbf{w}_{2} \mathbf{w}_{2}}^{2}\left(\mathbf{w}_{1}, \mathbf{w}_{2}, \varsigma\right)+\frac{1}{96 Q_{0}}-\frac{1}{2}\right]
$$

Employing the inverse generalized integral transform, we obtain 


$$
\mathscr{Q}\left(\mathbf{w}_{1}, \mathbf{w}_{2}, \varsigma\right)=\mathbb{J}^{-1}\left[\frac{\psi(\mathfrak{g})}{\phi(\mathfrak{g})} \mathscr{Q}\left(\mathbf{w}_{1}, \mathbf{w}_{2}, 0\right)+\frac{\chi+(1-\chi) \psi^{\chi}(\mathfrak{g})}{\psi^{\chi}(\mathfrak{g}) \mathbb{A}(\chi)} \rrbracket\left[\mathscr{Q}_{\mathbf{w}_{1} \mathbf{w}_{1}}^{2}\left(\mathbf{w}_{1}, \mathbf{w}_{2}, \varsigma\right)+Q_{\mathbf{w}_{2} \mathbf{w}_{2}}^{2}\left(\mathbf{w}_{1}, \mathbf{w}_{2}, \varsigma\right)+\frac{1}{96 Q_{0}}-\frac{1}{2}\right] .\right.
$$
find

Thanks to the generalized decomposition method, we

$$
\begin{aligned}
\mathbb{Q}_{0}\left(\mathbf{w}_{1}, \mathbf{w}_{2}, \varsigma\right) & =\mathbb{J}^{-1}\left[\frac{\psi(\mathfrak{I})}{\phi(\mathfrak{I})} \mathscr{Q}\left(\mathbf{w}_{1}, \mathbf{w}_{2}, 0\right)\right]=\mathbb{J}^{-1}\left[\frac{\psi(\mathfrak{I})}{\phi(\mathfrak{I})} \frac{1}{4} \sqrt{2\left(\mathbf{w}_{1}^{2}+\mathbf{w}_{2}^{2}\right)+\mathbf{w}_{2}+5}\right] \\
& =\frac{1}{4} \sqrt{2\left(\mathbf{w}_{1}^{2}+\mathbf{w}_{2}^{2}\right)+\mathbf{w}_{2}+5}
\end{aligned}
$$

Here, we surmise that the unknown function $\mathbb{Q}\left(\mathbf{w}_{1}, \mathbf{w}_{2}, \varsigma\right)$ can be written by an infinite series of the form

$$
Q\left(\mathbf{w}_{1}, \mathbf{w}_{2}, \varsigma\right)=\sum_{\ell=0}^{\infty} Q_{\ell}\left(\mathbf{w}_{1}, \mathbf{w}_{2}, \varsigma\right)
$$

Also, the nonlinearity $\mathscr{F}_{j}(\mathscr{Q}), j=1,2,3$, can be decomposed by an infinite series of polynomials represented by $\quad \mathscr{F}_{1}(Q)=Q_{\mathbf{w}_{1} \mathbf{w}_{1}}^{2}=\sum_{\ell=0}^{\infty} \mathscr{A}_{\ell}$ and $\mathscr{F}_{2}(Q)=\mathbb{Q}_{\mathbf{w}_{2} \mathbf{w}_{2}}^{2}=\sum_{\ell=0}^{\infty}$ $\mathscr{B}_{\ell}$ along with $\mathscr{F}_{3}(\hat{Q})=(1 / 96 Q)-(1 / 2)=\sum_{\ell=0}^{\infty} \mathscr{C}_{\ell}$ defined in (44) and (101), respectively.

For $\ell=0,1,2,3, \ldots$,

$$
\begin{aligned}
& \mathscr{Q}_{1}\left(\mathbf{w}_{1}, \mathbf{w}_{2}, \varsigma\right)=\mathbb{J}^{-1}\left[\frac{\chi+(1-\chi) \psi^{\chi}(\mathfrak{g})}{\psi^{\chi}(\mathfrak{I}) \mathbb{A}(\chi)} \mathbb{J}\left[\mathscr{A}_{0}+\mathscr{B}_{0}+\mathscr{D}_{0}\right]\right] \\
& =\frac{1}{24 \mathbb{A}(\chi)}\left(2\left(\mathbf{w}_{1}^{2}+\mathbf{w}_{2}^{2}+\mathbf{w}_{2}+5\right)\right)^{-1 / 2}\left[\frac{\chi \varsigma^{\chi}}{\Gamma(\chi+1)}+(1-\chi)\right] \text {, } \\
& \mathbb{Q}_{2}\left(\mathbf{w}_{1}, \mathbf{w}_{2}, \varsigma\right)=\mathbb{J}^{-1}\left[\frac{\chi+(1-\chi) \psi^{\chi}(\mathfrak{g})}{\psi^{\chi}(\mathfrak{g}) \mathbb{A}(\chi)} \mathbb{d}\left[\mathscr{A}_{1}+\mathscr{B}_{1}+\mathscr{D}_{1}\right]\right] \\
& =-\frac{1}{144 \mathbb{A}^{2}(\chi)}\left(2\left(\mathbf{w}_{1}^{2}+\mathbf{w}_{2}^{2}+\mathbf{w}_{2}+5\right)\right)^{-3 / 2}\left[\frac{\chi^{2} \varsigma^{2 \chi}}{\Gamma(2 \chi+1)}+2 \chi(1-\chi) \frac{\varsigma^{\chi}}{\Gamma(\chi+1)}+(1-\chi)^{2}\right], \\
& \mathbb{Q}_{3}\left(\mathbf{w}_{1}, \mathbf{w}_{2}, \varsigma\right)=\mathbb{J}^{-1}\left[\frac{\chi+(1-\chi) \psi^{\chi}(\mathfrak{g})}{\psi^{\chi}(\mathfrak{g}) \mathbb{A}(\chi)} \mathbb{J}\left[\mathscr{A}_{2}+\mathscr{B}_{2}+\mathscr{D}_{2}\right]\right] \\
& =\frac{1}{288 \mathbb{A}^{3}(\chi)}\left(2\left(\mathbf{w}_{1}^{2}+\mathbf{w}_{2}^{2}+\mathbf{w}_{2}+5\right)\right)^{-5 / 2}\left[\frac{\chi^{3} \varsigma^{3 \chi}}{\Gamma(3 \chi+1)}+3 \chi^{2}(1-\chi) \frac{\varsigma^{2 \chi}}{\Gamma(2 \chi+1)}+3 \chi(1-\chi)^{2} \frac{\varsigma^{\chi}}{\Gamma(\chi+1)}+(1-\chi)^{3}\right],
\end{aligned}
$$$$
\vdots
$$

The approximate solution for Example 4 is expressed as follows: 
TABle 4: Exact $\mathscr{Q}_{E}$ and approximate solution $\mathbb{Q}_{A}$ of $\mathbb{Q}\left(\mathbf{w}_{1}, \mathbf{w}_{2}, \varsigma\right)$ of Example 3 having absolute error when $\chi=1, \varsigma=10, r_{1}=-8 / 9$, and $\hbar=-1$ for different values of $\mathbf{w}_{1}$ and $\mathbf{w}_{2}$.

\begin{tabular}{|c|c|c|c|c|c|c|}
\hline$\left(\mathbf{w}_{1}, \mathbf{w}_{2}\right)$ & $Q_{E}$ sol. & $\mathbb{Q}_{\mathrm{CFD}}$ sol. & $\mathbb{Q}_{\mathrm{ABC}}$ sol. & VIMHP sol. [23] & Error $=E_{1}$ & Error $=E_{2}$ \\
\hline$(-450,450)$ & 224.939112 & 224.939000 & 224.938855 & 224.939112 & $9.8139 e-16$ & $9.8127 e-16$ \\
\hline$(-400,400)$ & 199.939313 & 199.939304 & 199.939295 & 199.939316 & $1.7688 e-15$ & $1.7679 e-15$ \\
\hline$(-300,300)$ & 149.939918 & 149.939822 & 149.939800 & 149.939925 & $7.4574 e-15$ & $7.4570 e-15$ \\
\hline$(-250,250)$ & 124.940402 & 124.940400 & 124.940398 & 124.940445 & $7.4465 e-15$ & $7.4456 e-15$ \\
\hline$(0,0)$ & 0.8531256 & 0.8531119 & 0.8531108 & 0.8531260 & $7.3883 e-3$ & $7.3880 e-3$ \\
\hline$(50,50)$ & 25.07696486 & 25.07696467 & 25.07696402 & 25.07696508 & $5.7070 e-11$ & $5.7004 e-11$ \\
\hline$(100,100)$ & 50.06964861 & 50.06964789 & 50.06964702 & 50.06964940 & $1.7965 e-12$ & $1.7953 e-12$ \\
\hline$(200,200)$ & 100.0661239 & 100.0661130 & 100.0661009 & 100.0661400 & $5.6333 e-14$ & $5.6300 e-14$ \\
\hline$(350,350)$ & 175.06457142 & 175.06457103 & 175.06457100 & 175.06457300 & $3.4370 e-15$ & $3.4355 e-15$ \\
\hline$(500,500)$ & 250.0639500 & 250.0635573 & 250.0634435 & 250.0639946 & $5.7707 e-16$ & $5.7700 e-16$ \\
\hline
\end{tabular}

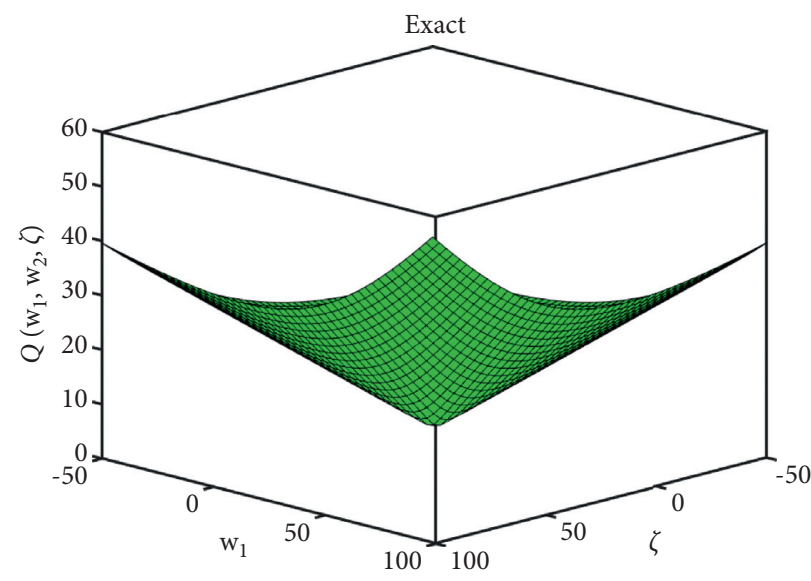

(a)

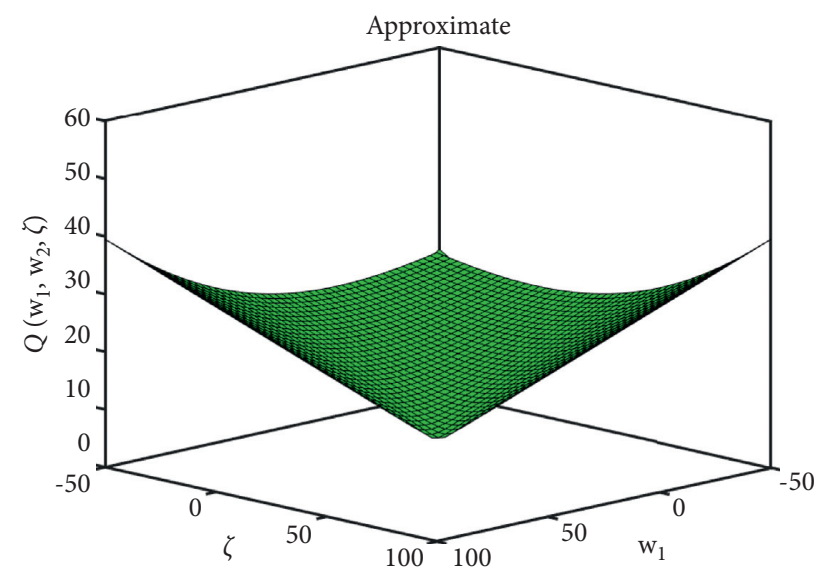

(b)

Figure 10: Three-dimensional illustration of exact and approximate solution of Example 4 when $\alpha=-1, \beta=1, r_{1}=48, \varsigma=0.01, \hbar=(1 / 96)$, and $\chi=1$.

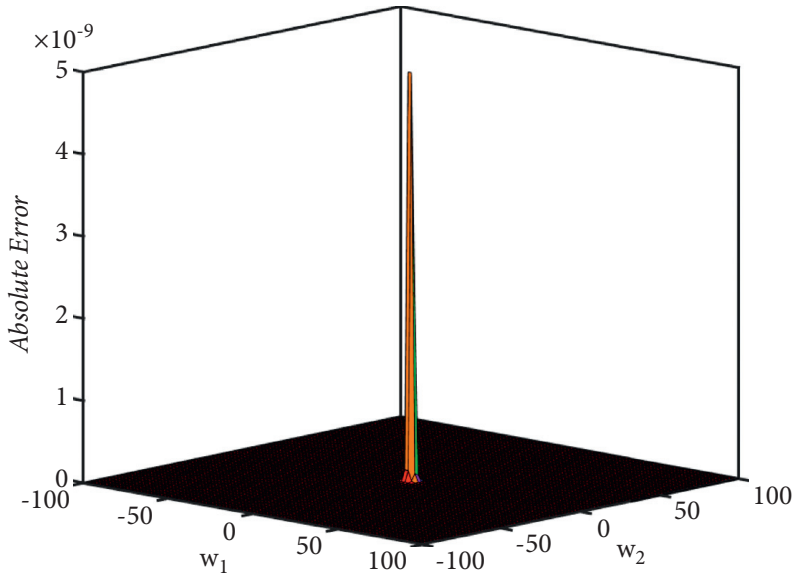

(a)

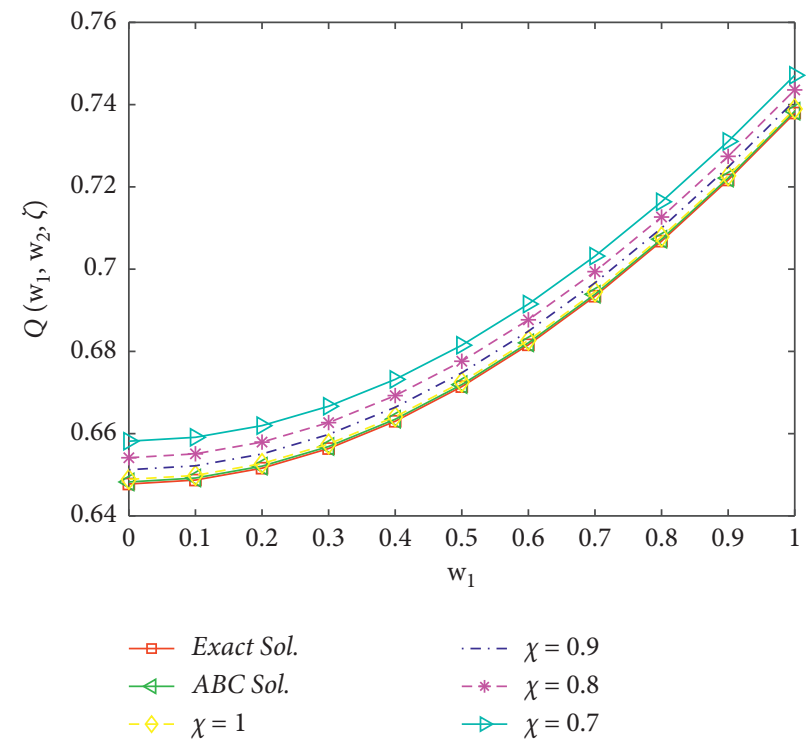

(b)

Figure 11: Three-dimensional illustration of the absolute error and two-dimensional view of multiple fractional orders for Example 4 when $\alpha=-1, \beta=1, r_{1}=48, \hbar=(1 / 96)$, and $\varsigma=0.01$. 


$$
\begin{aligned}
\mathbb{Q}\left(\mathbf{w}_{1}, \mathbf{w}_{2}, \varsigma\right)= & \Phi_{0}\left(\mathbf{w}_{1}, \mathbf{w}_{2}, \varsigma\right)+\Phi_{1}\left(\mathbf{w}_{1}, \mathbf{w}_{2}, \varsigma\right)+\Phi_{2}\left(\mathbf{w}_{1}, \mathbf{w}_{2}, \varsigma\right)+\Phi_{3}\left(\mathbf{w}_{1}, \mathbf{w}_{2}, \varsigma\right)+\cdots \\
= & \frac{1}{4} \sqrt{2\left(\mathbf{w}_{1}^{2}+\mathbf{w}_{2}^{2}\right)+\mathbf{w}_{2}+5}+\frac{1}{24 \mathbb{A}(\chi)}\left(2\left(\mathbf{w}_{1}^{2}+\mathbf{w}_{2}^{2}+\mathbf{w}_{2}+5\right)\right)^{-1 / 2}\left(\frac{\varsigma^{\chi}}{\Gamma(\chi+1)}+(1-\chi)\right) \\
& -\frac{1}{144 \mathbb{A}^{2}(\chi)}\left(2\left(\mathbf{w}_{1}^{2}+\mathbf{w}_{2}^{2}+\mathbf{w}_{2}+5\right)\right)^{-3 / 2}\left(\frac{\chi^{2} \varsigma^{2 \chi}}{\Gamma(2 \chi+1)}+2 \chi(1-\chi) \frac{\varsigma^{\chi}}{\Gamma(\chi+1)}+(1-\chi)^{2}\right) \\
& +\frac{1}{288 \mathbb{A}^{3}(\chi)}\left(2\left(\mathbf{w}_{1}^{2}+\mathbf{w}_{2}^{2}+\mathbf{w}_{2}+5\right)\right)^{-5 / 2}\left(\frac{\chi^{3} \varsigma^{3 \chi}}{\Gamma(3 \chi+1)}+3 \chi^{2}(1-\chi) \frac{\varsigma^{2 \chi}}{\Gamma(2 \chi+1)}+3 \chi(1-\chi)^{2} \frac{\varsigma^{\chi}}{\Gamma(\chi+1)}+(1-\chi)^{3}\right)+\cdots
\end{aligned}
$$

For $\chi=1$, we obtained the exact solution of Example 4 as

$$
Q\left(\mathbf{w}_{1}, \mathbf{w}_{2}, \varsigma\right)=\frac{1}{4} \sqrt{2\left(\mathbf{w}_{1}^{2}+\mathbf{w}_{2}^{2}\right)+\mathbf{w}_{2}+\frac{\varsigma}{3}+5} .
$$

Table 4 shows the analytical approximate solutions with some free parameters that are provided by the proposed technique. The comparison analysis is conducted with the VIMHP that predicts the preciseness of the suggested scheme due to their lower error. The analytical findings are extremely useful in deciphering the internal components of acts of nature. The exact and numerical solution for $\alpha=-1, \beta=1, r_{1}=48, \varsigma=0.01, \hbar=(1 / 96)$, and $\chi=1$ is shown in Figure 10.

Furthermore, the absolute error for the aforementioned assumptions is depicted in Figure 11(a).

Finally, Figure 11(b) represents the two-dimensional behavior of exact, approximate by Caputo, and approximate solutions derived by $\mathrm{ABC}$ fractional operators with varying fractional orders. These solutions have a distinctive characteristic that allows them to interact with other solutions derived by [23]. The proposed findings have particle-like geometries in their solutions. The synthesized trajectory is either a success or a descent from one asymptotic state to the next. The accuracy of the proposed method can be enhanced by increasing the recursive terms.

\section{Conclusion}

This article investigated the more general integral transform with the Adomian decomposition method. The Caputo and $\mathrm{ABC}$ fractional derivative operators have been implemented to deal with the biological population model. Several distinct solutions have been proposed with the assumptions of Malthusian law, Verhulst law, and porous media. Various representations were used to elucidate these solutions, which clarified the significant properties of the fractional models in consideration. Without any restrictive assumptions, discretization, or linearization, the proposed methodology locates the solutions. Elegance and originality have been invoked to describe our trajectory. Contrasting proposed findings to those acquired in earlier scholarly articles demonstrates the peculiarity of our solutions. The strategy's powerful and successful implementation is explored and validated in order to demonstrate its applicability to additional nonlinear evolution equations.

\section{Data Availability}

No data were used to support this study.

\section{Conflicts of Interest}

The authors declare that they have no conflicts of interest.

\section{Authors' Contributions}

S. Rashid provided the main ideas of the article, constructed the main algorithm, proved the convergence, and also submitted the article. R. Ashraf drafted the manuscript and provided two test examples with their illustration and uniqueness analysis. E. Bonyah provided the solution of Examples 3 and 4 and completed the final revision. All authors read and approved the final manuscript.

\section{References}

[1] K. B. Oldham and J. Panier, "The fractional calculus," Vol. 111 of Mathematics in science and engineering, Elsevier, Amsterdam, Netherlands, 1974.

[2] I. Podlubny, Fractional Differential Equations, Academic Press, San Diego, CA, USA, 1999.

[3] R. Hilfer, Applications of Fractional Calculus in Physics, Word Scientific, Singapore, 2000.

[4] A. A. Alderremy, K. M. Saad, J. F. Gómez-Aguilar, S. Aly, D. Kumar, and J. Singh, "New models of fractional blood ethanol and two-cell cubic autocatalator reaction equations," Mathematical Methods in the Applied Sciences, vol. 107, 2021.

[5] S. Aljhani, M. S. M. Noorani, K. M. Saad, and A. K. Alomari, "Numerical solutions of certain new models of the timefractional gray-Scott," J. Fun. Spaces, vol. 2021, Article ID 2544688, 12 pages, 2021.

[6] M. Caputo, Elasticita e Dissipazione, Zanichelli, Bologna, Italy, 1969.

[7] A. Atangana and D. Baleanu, "New fractional derivatives with nonlocal and non-singular kernel: theory and application to heat transfer model," Thermal Science, vol. 20, no. 2, pp. 763-769, 2016.

[8] A. A. Kilbas, H. M. Srivastava, and J. J. Trujillo, Theory and Applications of Fractional Differential Equations, Elsevier Science Limited, Amsterdam, Netherlands, 2006.

[9] M. Khader, K. M. Saad, Z. Hammouch, and D. Baleanu, “A spectral collocation method for solving fractional $\mathrm{KdV}$ and KdV-Burger's equations with non-singular kernel 
derivatives," Applied Numerical Mathematics, vol. 161, no. 4, 2020.

[10] K. M. Saad, M. Alqhtani, and M. Alqhtani, "Numerical simulation of the fractal-fractional reaction diffusion equations with general nonlinear," AIMS Mathematics, vol. 6, no. 4, pp. 3788-3804, 2021.

[11] A. J. M. Jawad, "New Exact Solutions of nonlinear partial differential equations using Tan-Cot function method," Studies in Mathematical Sciences, vol. 5, no. 2, pp. 12-24, 2012.

[12] W. Li and Y. Pang, "Application of Adomian decomposition method to nonlinear systems," Advances in Difference Equations, vol. 2020, no. 1, p. 67, 2020.

[13] J.-H. He, "Homotopy perturbation technique," Computer Methods in Applied Mechanics and Engineering, vol. 178, no. 3-4, pp. 257-262, 1999.

[14] M. Turkyilmazoglu, "A note on the homotopy analysis method," Applied Mathematics Letters, vol. 23, no. 10, pp. 1226-1230, 2010.

[15] G. Hariharan and K. Kannan, "Review of wavelet methods for the solution of reaction-diffusion problems in science and engineering," Applied Mathematical Modelling, vol. 38, no. 3, pp. 799-813, 2014.

[16] N. Maarouf, H. Maadan, and K. Hilal, "Lie symmetry analysis and explicit solutions for the time-fractional regularized Long-Wave equation," International Journal of Differential Equations, vol. 2021, Article ID 6614231, 11 pages, 2021.

[17] M. E. Gurtin and R. C. Maccamy, "On the diffusion of biological population," Mathematical Biosciences, vol. 33, no. 35-49, 1977.

[18] W. S. C. Gurney and R. M. Nisbet, "The regulation of inhomogeneous populations," Journal of Theoretical Biology, vol. 52, no. 2, pp. 441-457, 1975.

[19] Y.-G. Lu, "Hölder estimates of solutions of biological population equations," Applied Mathematics Letters, vol. 13, no. 6, pp. 123-126, 2000.

[20] J. Bear, Dynamics of Fluids in Porous Media, Courier Corporation, Chelmsford, MA, USA, 2013.

[21] A. Okubo and S. A. Levin, "Diffusion and ecological problems," Math Models, Springer, Berlin, Germany, 1980.

[22] S. Rashid, K. Tul Kubra, and S. Ullah, "Fractional spatial diffusion of a biological population model via a new integral transform in the settings of power and Mittag-Leffler nonsingular kernel," Physica Scripta, vol. 96, no. 11, Article ID 114003, 2021.

[23] M. Zellal and K. Belghaba, “'An accurate algorithm for solving biological population model by the variational iteration method using He's polynomials," Arab Journal of Basic and Applied Sciences, vol. 25, no. 3, pp. 142-149, 2018.

[24] J. Singh, "Analysis of fractional blood alcohol model with composite fractional derivative," Chaos, Solitons \& Fractals, vol. 140, Article ID 110127, 2020.

[25] P. A. Naik, J. Zu, and K. M. Owolabi, "Global dynamics of a fractional order model for the transmission of HIV epidemic with optimal control," Chaos, Solitons, and Fractals, vol. 138, Article ID 109826, 2020.

[26] A. Atangana and E. Alabaraoye, "Solving a system of fractional partial differential equations arising in the model of $\mathrm{HIV}$ infection of $\mathrm{CD}^{+}$cells and attractor one-dimensional Keller-Segel equations," Advances in Difference Equations, vol. 2013, p. 94, 2013.

[27] P. A. Naik, "Global dynamics of a fractional-order SIR epidemic model with memory," International Journal of Biomathematics, vol. 13, Article ID 2050071, 2020.
[28] S. Noeiaghdam and D. Sidorov, "Caputo-Fabrizio fractional derivative to solve the fractional model of energy supplydemand system," Mathematical Modelling of Engineering Problems, vol. 7, no. 3, pp. 359-367, 2020.

[29] S. Rashid, A. Khalid, S. Sultana, Z. Hammouch, R. Shah, and A. M. Alsharif, "A novel analytical view of time-fractional Korteweg-De Vries equations via a new integral transform," Symmetry, vol. 13, no. 7, p. 1254, 2021.

[30] S. Rashid, Z. Hammouch, H. Aydi, A. G. Ahmad, and A. M. Alsharif, "Novel computations of the time-fractional Fisher's model via generalized fractional integral operators by means of the Elzaki transform," Fractal and Fractional, vol. 5, no. 3, p. 94, 2021.

[31] S. Rashid, K. T. Kubra, and J. L. G. Guirao, "Construction of an approximate analytical solution for multi-dimensional fractional Zakharov-Kuznetsov equation via Aboodh Adomian decomposition method," Symmetry, vol. 13, no. 8, p. 1542, 2021.

[32] H. Jafari, "A new general integral transform for solving integral equations," Journal of Advanced Research, vol. 32, pp. 133-138, 2021.

[33] L. Debnath and D. Bhatta, Integral Transforms and Their Applications, CRC Press, Boca Raton, FL, USA, 2014.

[34] F. Jarad and T. Abdeljawad, "A modified Laplace transform for certain generalized fractional operators," Results in Nonlinear Analysis, vol. 1, no. 2, pp. 88-98, 2018.

[35] G. K. Watugala, "Sumudu transform: a new integral transform to solve differential equations and control engineering problems," International Journal of Mathematical Education in Science \& Technology, vol. 24, no. 1, pp. 35-43, 1993.

[36] K. S. Aboodh, "The new integral transform Aboodh transform," Global Journal of Pure and Applied Mathematics, vol. 9, no. 35-43, 2013.

[37] S. A. P. Ahmadi, H. Hosseinzadeh, and A. Y. Cherati, "A new integral transform for solving higher order linear ordinary differential equations," Nonlinear Dynamics and Systems Theory, vol. 19, no. 2, pp. 243-252, 2019.

[38] S. A. P. Ahmadi, H. Hosseinzadeh, and A. Y. Cherati, "A new integral transform for solving higher order linear ordinary Laguerre and Hermite differential equations," International Journal of Applied and Computational Mathematics, vol. 5, no. 142, 2019.

[39] T. M. Elzaki, "The new integral transform Elzaki Transform," Global Journal of Pure and Applied Mathematics, vol. 7, no. 1, pp. 57-64, 2011.

[40] Z. H. Khan and W. A. Khan, "N-transform properties and applications," NUST Journal of Engineering Sciences, vol. 1, no. 1, pp. 127-133, 2008.

[41] M. M. Abdelrahim Mahgoub, "The new integral transform mohand transform," Advances in Theoretical and Applied Mathematics, vol. 12, no. 2, pp. 113-120, 2017.

[42] M. M. Abdelrahim Mahgoub, "The new integral transform sawi transform," Advances in Theoretical and Applied Mathematics, vol. 14, no. 1, pp. 81-87, 2019.

[43] H. Kamal and A. Sedeeg, "The new integral transform Kamal transform," Advances in Theoretical and Applied Mathematics, vol. 11, no. 4, pp. 451-458, 2016.

[44] H. Kim, "On the form and properties of an integral transform with strength in integral transforms," Far East Journal of Mathematical Sciences, vol. 102, no. 11, pp. 2831-2844, 2017.

[45] H. Kim, "The intrinsic structure and properties of Laplacetyped integral transforms," Mathematical Problems in Engineering, vol. 2017, Article ID 1762729, 8 pages, 2017. 
[46] M. Meddahi, H. Jafari, and M. N. Ncube, "New general integral transform via Atangana-Baleanu derivatives," Advances in Difference Equations, vol. 2021, no. 1, p. 385, 2021.

[47] A. Atangana and I. Koca, "Chaos in a simple nonlinear system with Atangana-Baleanu derivatives with fractional order," Chaos, Solitons \& Fractals, vol. 89, pp. 447-454, 2016.

[48] M. Yavuz and T. Abdeljawad, "Nonlinear regularized longwave models with a new integral transformation applied to the fractional derivative with power and Mittag-Leffler kernel," Advances in Differential Equations, vol. 2020, p. 367, 2020.

[49] A. Bokhari, D. Baleanu, and R. Belgacema, "Application of Shehu transform to Atangana-Baleanu derivatives," The Journal of Mathematics and Computer Science, vol. 20, pp. 101-107, 2020.

[50] M. G. Mittag-Leffler, "Sur la nouvelle fonction $\mathrm{Ea}(\mathrm{x})$," Comptes Rendus de l'Académie des Sciences, vol. 2, no. 1003, 1903.

[51] I. L. El-Kalla, "Convergence of the Adomian method applied to a class of nonlinear integral equations," Applied Mathematics Letters, vol. 21, no. 4, pp. 372-376, 2008.

[52] G. Adomian and R. Rach, "Modified adomian polynomials," Mathematical and Computer Modelling, vol. 24, no. 11, pp. 39-46, 1996.

[53] P. Roul, "Application of homotopy perturbation method to biological population model," Applications and Mathematics: An International Journal, vol. 10, pp. 1369-1378, 2010.

[54] F. Shakeri and M. Dehghan, "Numerical solution of a biological population model using He's variational iteration method," Computers \& Mathematics with Applications, vol. 54, no. 7-8, pp. 1197-1209, 2007. 